\title{
LONG TIME PROPAGATION AND CONTROL ON SCARRING FOR PERTURBED QUANTIZED HYPERBOLIC TORAL AUTOMORPHISMS
}

\author{
Jean-Marc Bouclet* Stephan De Bièvre ${ }^{\dagger}$ \\ Université de Lille 1 \\ UMR CNRS 8524, \\ 59655 Villeneuve d'Ascq
}

6th November 2018

\begin{abstract}
We show that on a suitable time scale, logarithmic in $\hbar$, the coherent states on the twotorus, evolved under a quantized perturbed hyperbolic toral automorphism, equidistribute on the torus. We then use this result to obtain control on the possible strong scarring of eigenstates of the perturbed automorphisms by periodic orbits. Our main tool is an adapted Egorov theorem, valid for logarithmically long times.
\end{abstract}

\section{Introduction}

One of the main results in quantum chaos is the Schnirelman theorem. It states that, if a quantum system has an ergodic classical limit, then almost all sequences of its eigenfunctions converge, in the classical limit, to the Liouville measure on the relevant energy surface [7, 15, 20, 24]. It is natural to wonder if the result holds for all sequences (a statement commonly referred to as "unique quantum ergodicity"). This has been proven to be true for the (Hecke) eigenfunctions of the Laplace-Beltrami operator of a certain class of constant negative curvature surfaces [17 and has been conjectured to be true for all such surfaces 19. It also has been proven to be wrong for quantized toral automorphisms in [11. In that case, sequences of eigenfunctions exist with a semiclassical limit having up to half of its weight supported on a periodic orbit of the dynamics. This phenomenon is referred to as (strong) scarring. In [5, 12, it is shown that this last result is optimal: if a measure is obtained as the limit of eigenfunctions then its pure point component can carry at most half of its total weight.

Except for the Schnirelman theorem, which holds in very great generality, all cited results are proven by exploiting to various degrees special algebraic or number theoretic properties of the systems studied. It is one of the major challenges in the field to device proofs and obtain results that use only assumptions on the dynamical properties of the underlying classical Hamiltonian system, such as ergodicity, mixing or exponential mixing, the Anosov property, etc. without relying on special algebraic properties.

\footnotetext{
*Jean-Marc.Bouclet@math.univ-lille1.fr

†Stephan.De-Bievre@math.univ-lille1.fr
} 
It is argued in 4, 5, 12 for example, that this will require a good control on the quantum dynamics for times that go to infinity (at least) logarithmically as the semiclassical parameter $\hbar$ goes to zero: $t \geq k_{-} \ln \hbar$ for some constant $k_{-}>0$. It is well known that such control is in general hard to obtain especially since a good lower bound on $k_{-}$is needed. In this paper, we concentrate on the quantized perturbed hyperbolic automorphisms of the $2 d$-torus, which are known to be Anosov systems classically. For those systems, we first prove an Egorov theorem valid for times proportional to $\ln \hbar$, with an explicit control on the proportionality constant $k_{-}$(Theorem 3.1). This result is obtained by adapting the techniques of [8]. We then combine this result with recent sharp estimates on the exponential mixing of the classical dynamics 3 to study the long time evolution of evolved coherent states (Theorem 4.7), showing that on a sufficiently long logarithmic time scale, those evolved coherent states equidistribute on the torus. Roughly, the result is that for all $f \in C^{\infty}\left(\mathbb{T}^{2}\right)$,

$$
Q(f, t, \hbar) \equiv\left\langle U_{\epsilon}^{t} \varphi_{\hbar, \kappa}^{a}, O p^{W}(f) U_{\epsilon}^{t} \varphi_{\hbar, \kappa}^{a}\right\rangle_{\mathcal{H}_{\hbar}(\kappa)}-\int_{\mathbb{T}^{2}} f(x) \mathrm{d} x \rightarrow 0, \quad \hbar \rightarrow 0
$$

for times

$$
k_{-} \ln \hbar \leq t \leq k_{+} \ln \hbar, \quad 0 \leq k_{-} \leq k_{+} .
$$

Here $U_{\epsilon}$ is the unitary quantum dynamical evolution operator, $O p^{W}(f)$ is the Weyl quantization of $f$, and $\varphi_{\hbar, \kappa}^{a}$ is a coherent state at the point $a$ of the two-torus $\mathbb{T}^{2}$. For detailed definitions, we refer to the following sections. This result generalizes results obtained in 4 ] for unperturbed hyperbolic automorphisms. To prove it, we prove an estimate of the type

$$
\begin{aligned}
Q(f, t, \hbar) \leq\left|\left\langle\varphi_{\hbar, \kappa}^{a},\left(U_{\epsilon}^{-t} O p^{W}(f) U_{\epsilon}^{t}-O p^{W}\left(f \circ \Phi_{\epsilon}^{t}\right)\right) \varphi_{\hbar, \kappa}^{a}\right\rangle_{\mathcal{H}_{\hbar}(\kappa)}\right|+ \\
\\
\quad\left|\left\langle\varphi_{\hbar, \kappa}^{a}, O p^{W}\left(f \circ \Phi_{\epsilon}^{t}\right)\right) \varphi_{\hbar, \kappa}^{a}\right\rangle_{\mathcal{H}_{\hbar}(\kappa)}-\int_{\mathbb{T}^{2}} f(x) \mathrm{d} x \mid \\
\leq \epsilon_{1}\left(\hbar e^{\gamma_{q} t}\right)+\epsilon_{2}\left(\hbar^{-1} e^{-\gamma_{c} t}\right) .
\end{aligned}
$$

Here $\epsilon_{1}$ and $\epsilon_{2}$ are functions tending to zero when their argument does. The first term comes from the error term in the Egorov theorem, whereas the second one involves a classical mixing rate $\gamma_{c}$. It is obvious that this estimate leads to the result only if $\gamma_{q}<\gamma_{c}$. One therefore needs $\gamma_{c}$ to be large (fast mixing) and $\gamma_{q}$ to be small. Sharp results on the classical mixing rates of Anosov systems are hard to come by, but for some Anosov maps, among which the perturbed toral automorphisms that are the subject of this paper, such results have become available recently [3]. The remaining difficulty resides therefore in controlling the exponent in the error in the Egorov theorem. This is dealt with in the next section.

We note that, although we prove the Egorov theorem for systems on the $2 d$-torus, we only prove the result above in full generality for $d=1$. Indeed, denoting for arbitrary $d$ by $\Gamma_{\min }$ and $\Gamma_{\max }$ the smallest and largest Lyapounov exponents of the system, we prove in Section 2 that, essentially, $\gamma_{q}=\frac{3}{2} \Gamma_{\max }$. On the other hand, the available estimates on the classical mixing rate 3 yield in our context here $\gamma_{c}=2 \Gamma_{\min }$. Of course, when $d=1, \Gamma_{\max }=\Gamma_{\min }$ and we have $\gamma_{q}<\gamma_{c}$ as needed. This leads to 1.1 For $d>1$, on the other hand, our proof of (1.1) still goes through, but only under an artificial "pinching" condition on the Lyapounov exponents of the type $3 \Gamma_{\max }<4 \Gamma_{\min }$.

As an application of the above result, we finally show how to use the information obtained on the evolved coherent states in combination with the basic strategy of [5, 6] to gain some control on the scarring of eigenfunctions (Theorem 4.9 Corollary 4.10). Roughly speaking, we show that if a sequence of eigenfunctions of a quantized perturbed hyperbolic toral automorphism converges to a delta measure on a finite union of periodic orbits, then it must do so slowly. An improvement 
on this result (basically, on how slowly) has been announced recently in 13. We don't expect this result to be optimal: indeed, it is expected, as in the case of unperturbed automorphisms, that sequences of eigenfunctions can not concentrate completely on periodic orbits, no matter how slowly. Proving this would involve controlling the quantum dynamics for longer times than we are currently able to do.

A result somewhat analogous to our result on the evolution of coherent states was recently obtained for the long time evolution of Lagrangian states on compact Riemannian manifolds of negative curvature 21]. It should however be noted that such a result does not require any control on the proportionality constant preceding $\ln \hbar$ so that no precise control on either the mixing rate or the exponent in the error term of the Egorov theorem are needed in that case. We suspect that in situations were such control can be obtained, our present strategy will allow to control both coherent state evolution and strong scarring.

A related result for the eigenfunctions of Laplace-Beltrami operators on compact, negatively curved Riemannian manifolds is proven using a different strategy in [1]: it is shown there that (under a suitable technical condition that may or may not hold) such eigenfunctions can not concentrate on sets of small topological entropy (and therefore on periodic orbits).

\section{Weyl quantization and Egorov Theorem}

The purpose of this section is to recall (as compactly as possible) some properties of the Weyl quantization on $\mathbb{T}^{2 d}:=(\mathbb{R} / \mathbb{Z})^{2 d}$ as well as on $\mathbb{R}^{2 d}$, for $d \geq 1$. More specifically, we want to state a semi-classical version of the Egorov Theorem in the case of $\mathbb{T}^{2 d}$. The latter is of course well known for $\mathbb{R}^{2 d}$ but it requires a proof for $\mathbb{T}^{2 d}$ all the more so as we need a rather explicit version of this theorem for the applications we have in mind in this paper.

The Weyl quantization on $\mathbb{R}^{2 d}$ can be defined as the linear map

$$
f \in \mathcal{B}\left(\mathbb{R}^{2 d}\right) \mapsto O p^{W}(f) \in \mathcal{L}\left(L^{2}\left(\mathbb{R}^{d}\right)\right)
$$

where $O p^{W}(f)$ is the operator (belonging a priori to $\mathcal{L}\left(\mathcal{S}\left(\mathbb{R}^{d}\right), \mathcal{S}^{\prime}\left(\mathbb{R}^{d}\right)\right)$ ) with Schwartz kernel

$$
K_{f}\left(q_{1}, q_{2}\right)=(2 \pi)^{-d} \int_{\mathbb{R}^{d}} \exp \left(i\left(q_{1}-q_{2}\right) \cdot p\right) f\left(\frac{q_{1}+q_{2}}{2}, \hbar p\right) \mathrm{d} p .
$$

Here $\mathcal{B}\left(\mathbb{R}^{2 d}\right)$ is the set of smooth functions $f$ on $\mathbb{R}^{2 d}$ such that $\partial^{\gamma} f$ is bounded for all $\gamma \in \mathbb{N}^{2 d}$, thus the above integral has to be understood in the sense of oscillatory integrals [16, 22, 18, 14, but it is of course a usual Lebesgue integral if $f$ decays fast enough at infinity. The fact that $O p^{W}(f)$ can be considered as a bounded operator on $L^{2}\left(\mathbb{R}^{d}\right)$ follows from the Calderòn-Vaillancourt theorem [16, 22, 18, 14, which states the existence of $C>0$ and $\bar{d}>0$ such that

$$
\left\|O p^{W}(f) \psi\right\|_{L^{2}\left(\mathbb{R}^{d}\right)} \leq C \sup _{|\gamma| \leq \bar{d}}\left\|\partial^{\gamma} f\right\|_{L^{\infty}\left(\mathbb{R}^{2 d}\right)}\|\psi\|_{L^{2}\left(\mathbb{R}^{d}\right)}, \quad \forall f \in \mathcal{B}\left(\mathbb{R}^{2 d}\right), \psi \in \mathcal{S}\left(\mathbb{R}^{d}\right) .
$$

It is moreover well known that $O p^{W}(f)$ maps the Schwartz space $\mathcal{S}\left(\mathbb{R}^{d}\right)$ continuously into itself and that $O p^{W}(f)^{*}=O p^{W}(\bar{f})$, thus $O p^{W}(f)$ can be considered as a continuous operator on $\mathcal{S}^{\prime}\left(\mathbb{R}^{d}\right)$ too. Note also that $O p^{W}(f)$ is self-adjoint on $L^{2}\left(\mathbb{R}^{d}\right)$ when $f$ is real valued.

The Weyl quantization on $\mathbb{T}^{2 d}$ is obtained by restricting $O p^{W}(f)$ to certain subspaces of $\mathcal{S}^{\prime}\left(\mathbb{R}^{d}\right)$ when $f \in C^{\infty}\left(\mathbb{T}^{2 d}\right)$ (i.e. is $\mathbb{Z}^{2 d}$ periodic). The construction is as follows (see [7 for more details). For any $\xi=\left(\xi_{q}, \xi_{p}\right) \in \mathbb{R}^{2 d}$, the phase space translation operator $U_{\hbar}(\xi)$ is defined by

$$
U_{\hbar}(\xi) \psi(q)=\psi\left(q-\xi_{q}\right) \exp \frac{i}{\hbar}\left(\xi_{p} \cdot q-\frac{\xi_{q} \cdot \xi_{p}}{2}\right), \quad \psi \in \mathcal{S}\left(\mathbb{R}^{d}\right)
$$


and is clearly a unitary operator on $L^{2}\left(\mathbb{R}^{d}\right)$. One easily checks that

$$
U_{\hbar}(\xi)=O p^{W}\left(\chi_{\xi}\right), \quad \chi_{\xi}(q, p)=\exp \frac{i}{\hbar}\left(q \cdot \xi_{p}-p \cdot \xi_{q}\right)
$$

and that the following Weyl-Heisenberg relations hold for all $\xi, \eta \in \mathbb{R}^{2 d}$

$$
U_{\hbar}(\xi) U_{\hbar}(\eta)=\exp \frac{i}{2 \hbar} \omega(\eta, \xi) U_{\hbar}(\xi+\eta),
$$

with $\omega$ the symplectic form defined by $\omega(\xi, \eta)=\xi_{q} \cdot \eta_{p}-\xi_{p} \cdot \eta_{q}$. This relation shows in particular that, if $n, m \in \mathbb{Z}^{2 d}, U_{\hbar}(n)$ and $U_{\hbar}(m)$ commute if and only if there exists $N \in \mathbb{N}$ such that

$$
2 \pi \hbar N=1
$$

Since $U_{\hbar}(\xi)$ acts naturally on $\mathcal{S}^{\prime}(\mathbb{R})$, we can introduce for any $\kappa \in[0,2 \pi)^{2 d}$ the space

$$
\mathcal{H}_{\hbar}(\kappa)=\left\{\psi \in \mathcal{S}^{\prime}\left(\mathbb{R}^{d}\right) \mid U_{\hbar}(n) \psi=e^{i \omega(\kappa, n)+i \frac{n_{q} \cdot n_{p}}{2 \hbar}} \psi, \quad \forall n=\left(n_{q}, n_{p}\right) \in \mathbb{Z}^{2 d}\right\}
$$

and it turns out that $\mathcal{H}_{\hbar}(\kappa)$ is of dimension $N^{d}$ if (2.4) holds (0 otherwise) with the basis

$$
\psi_{r}^{\kappa}(q)=N^{-\frac{d}{2}} \sum_{k \in \mathbb{Z}^{2 d}} e^{i \kappa_{p} \cdot k} \delta_{0}\left(q-k-\frac{r}{N}-\frac{\kappa_{q}}{2 \pi N}\right), \quad r \in\{0, \cdots, N-1\}^{d} .
$$

The latter is proven in [7] as well as the existence of a unique scalar product on each $\mathcal{H}_{\hbar}(\kappa)$ making the above basis orthonormal and $U_{\hbar}(n / N)$ unitary for all $n \in \mathbb{Z}^{2 d}$. The Weyl quantization on $\mathbb{T}^{2 d}$ is then defined by

$$
f \in C^{\infty}\left(\mathbb{T}^{2 d}\right) \mapsto O p^{W}(f)_{\mid \mathcal{H}_{\hbar}(\kappa)}
$$

This is indeed a mapping from $C^{\infty}\left(\mathbb{T}^{2 d}\right)$ to $\mathcal{L}\left(\mathcal{H}_{\hbar}(\kappa)\right)$, i.e. $\mathcal{H}_{\hbar}(\kappa)$ is stable under $O p^{W}(f)$, since one can easily check that for any $\mathbb{Z}^{2 d}$ periodic function $f$

$$
O p^{W}(f)=\sum_{n \in \mathbb{Z}^{2 d}} f_{n} U_{\hbar}(n / N)
$$

if $f(x)=\sum_{n} f_{n} e^{2 i \pi \omega(x, n)}, x \in \mathbb{R}^{2 d}$. Let us emphasize that the spaces $\mathcal{H}_{\hbar}(\kappa)$ are very natural in view of the following direct integral decomposition 7$]$

$$
L^{2}\left(\mathbb{R}^{d}\right) \simeq(2 \pi)^{-2 d} \int_{[0,2 \pi)^{2 d}}^{\oplus} \mathcal{H}_{\hbar}(\kappa) \mathrm{d} \kappa
$$

in which the operators $O p^{W}(f)_{\mid \mathcal{H}_{\hbar}(\kappa)}$ are the fibers of $O p^{W}(f)$ for this decomposition.

To streamline the discussion we will write both quantizations on $\mathbb{R}^{2 d}$ and $\mathbb{T}^{2 d}$ under a single form. From now on, $\mathcal{M}$ will denote either $\mathbb{R}^{2 d}$ or $\mathbb{T}^{2 d}$. The Weyl quantization on $\mathcal{M}$ can then be defined as the map

$$
f \in \mathcal{B}(\mathcal{M}) \rightarrow O p^{W}(f) \in \mathcal{L}(\mathcal{H})
$$

where $\mathcal{B}(\mathcal{M})$ is either $\mathcal{B}\left(\mathbb{R}^{2 d}\right)$ or $C^{\infty}\left(\mathbb{T}^{2 d}\right)$ and $\mathcal{H}$ is either $L^{2}\left(\mathbb{R}^{d}\right)$ or $\mathcal{H}_{\hbar}(\kappa)$ (we omit the $\hbar, \kappa$ dependence in the notations). In order to write $O p^{W}(f)$ in a unified way, we need to introduce the symplectic Fourier transform $\mathcal{F}$ on $\mathcal{M}$ defined by

$$
\mathcal{F} f(\xi)=\int_{\mathcal{M}} \exp (i \omega(\xi, x)) f(x) \mathrm{d} x
$$


where $\xi=\left(\xi_{q}, \xi_{p}\right)^{1}$ belongs to $\mathcal{M}^{*}=\mathbb{R}^{2 d}$ if $\mathcal{M}=\mathbb{R}^{2 d}$ and $(2 \pi \mathbb{Z})^{2 d}$ if $\mathcal{M}=\mathbb{T}^{2 d}$. Then the following inversion formula holds

$$
f(x)=\int_{\mathcal{M}^{*}} \exp i \omega(x, \xi) \mathcal{F} f(\xi) \mathrm{d} \nu(\xi)
$$

with $\mathrm{d} \nu=(2 \pi)^{-2 d} \times$ the Lebesgue measure (resp. $\left.\sum_{\mathbb{Z}^{2 d}} \delta_{2 \pi n}\right)$ if $\mathcal{M}^{*}=\mathbb{R}^{2 d}\left(\right.$ resp. $\left.(2 \pi \mathbb{Z})^{2 d}\right)$ and the Weyl quantization can easily be seen to be

$$
O p^{W}(f)=\int_{\mathcal{M}^{*}} U_{\hbar}(\hbar \xi) \mathcal{F} f(\xi) \mathrm{d} \nu(\xi) .
$$

Of course, when $\mathcal{M}=\mathbb{R}^{2 d}$, all the integrals must be understood in the weak sense (in (2.6) we use the fact that $\left\langle U_{\hbar}(\hbar \xi) \psi_{1}, \psi_{2}\right\rangle$ belongs to $\mathcal{S}\left(\mathbb{R}_{\xi}^{2 d}\right)$ if $\left.\psi_{1}, \psi_{2} \in \mathcal{S}\left(\mathbb{R}^{d}\right)\right)$. Note also the existence of $C, \bar{d}$ such that, if $\|.\|_{\infty}$ denotes the $L^{\infty}$ norm on $\mathcal{M}$,

$$
\left\|O p^{W}(f)\right\|_{\mathcal{H} \rightarrow \mathcal{H}} \leq C \sup _{|\gamma| \leq \bar{d}}\left\|\partial^{\gamma} f\right\|_{\infty}, \quad \forall f \in \mathcal{B}(\mathcal{M}) .
$$

This comes from (2.2) if $\mathcal{M}=\mathbb{R}^{2 d}$ and from the unitarity of $U_{\hbar}(n / N)$ combined with the elementary estimate $\sum_{n}\left|f_{n}\right| \leq C \sup _{|\gamma| \leq 2 d+1}\left\|\partial^{\gamma} f\right\|_{\infty}$ when $\mathcal{M}=\mathbb{T}^{2 d}$.

This completes the definition of the Weyl quantization on $\mathcal{M}$. Regarding the composition of the corresponding operators, we have the

Proposition 2.1. There exists a bilinear map $(f, g) \mapsto f \# g$ from $\mathcal{B}(\mathcal{M})^{2}$ to $\mathcal{B}(\mathcal{M})$ such that

$$
O p^{W}(f) O p^{W}(g)=O p p^{W}(f \# g) .
$$

The function $f \# g$ has a full asymptotic expansion in powers of $\hbar$, meaning that for all integers $J$

$$
f \# g=\sum_{j<J} \hbar^{j} f \#_{j} g+\hbar^{J} r_{J}^{\hbar}(f, g)
$$

where $f \#_{j} g=\sum_{|\alpha+\beta|=j} \Gamma(\alpha, \beta) \partial_{q}^{\alpha} \partial_{p}^{\beta} f \partial_{q}^{\beta} \partial_{p}^{\alpha} g$, with $\Gamma(\alpha, \beta)^{-1}=(-1)^{\alpha} \alpha ! \beta !(2 i)^{|\alpha+\beta|}$ and for all $\gamma \in \mathbb{N}^{2 d}$

$$
\left\|\partial^{\gamma} r_{J}^{\hbar}(f, g)\right\|_{\infty} \leq \frac{C_{d}^{J+|\gamma|}}{J !} \sup _{\left|\gamma_{1}\right| \leq J+|\gamma|+\tilde{d}}\left\|\partial^{\gamma_{1}} f\right\|_{\infty} \sup _{\left|\gamma_{2}\right| \leq J+|\gamma|+\tilde{d}}\left\|\partial^{\gamma_{2}} g\right\|_{\infty}, \quad 0<\hbar \leq 1 .
$$

for some constants $C_{d}, \tilde{d}$ depending only on $d$.

Note that $\left(. \#_{j}.\right)$ is symmetric (resp. skew symmetric) for $j$ even (resp. odd) and that

$$
g \#_{1} f-f \#_{1} g=-i\left(\nabla_{p} g \cdot \nabla_{q} f-\nabla_{q} g \cdot \nabla_{p} f\right)=-i\{g, f\} .
$$

Proof. This result is well known if $\mathcal{M}=\mathbb{R}^{2 d}$ (see for instance the appendix of [8] for a simple proof). We briefly sketch the proof in the case $\mathcal{M}=\mathbb{T}^{2 d}$. Using (2.3) and (2.6), we have

$$
\begin{aligned}
O p^{W}(f) O p^{W}(g) & =\int_{\mathcal{M}^{*}} \int_{\mathcal{M}^{*}} e^{i \hbar \omega(\eta, \xi) / 2} U_{\hbar}(\hbar(\xi+\eta)) \mathcal{F} f(\xi) \mathcal{F} g(\eta) \mathrm{d} \nu(\xi) \mathrm{d} \nu(\eta) \\
& =\int_{\mathcal{M}^{*}} U_{\hbar}(\hbar \xi)\left(\int_{\mathcal{M}^{*}} e^{i \hbar \omega(\eta-\xi, \eta) / 2} \mathcal{F} f(\xi-\eta) \mathcal{F} g(\eta) \mathrm{d} \nu(\eta)\right) \mathrm{d} \nu(\xi) .
\end{aligned}
$$

\footnotetext{
${ }^{1}$ Throughout this paper, $x$ will denote the running point of $\mathcal{M}$ and $\xi$ the one of $\mathcal{M}^{*}$, unlike the usual notation of microlocal analysis where $(x, \xi)$ is the running point of $\mathbb{R}^{2 d}$.
} 
Expanding $e^{i \hbar \omega(\eta-\xi, \eta) / 2}$ by the Taylor formula, we get the expansion of $f \# g$ with a remainder $r_{J}=r_{J}^{\hbar}(f, g)$ defined by its Fourier transform as follows

$$
\mathcal{F} r_{J}(\xi)=\frac{(-2 i)^{-J}}{(J-1) !} \int_{0}^{1}(1-t)^{J-1} \int_{\mathcal{M}^{*}} e^{i t \hbar \omega(\eta-\xi, \eta)} \omega(\eta-\xi, \eta)^{J} \mathcal{F} f(\xi-\eta) \mathcal{F} g(\eta) \mathrm{d} \nu(\eta) \mathrm{d} t .
$$

Since $\left|\xi^{\gamma} \mathcal{F} r_{J}(\xi)\right|=\left|\mathcal{F} \partial \widehat{\gamma} r_{J}(\xi)\right|$, with $\widehat{\gamma}=\left(\gamma_{p}, \gamma_{q}\right)$ if $\gamma=\left(\gamma_{q}, \gamma_{p}\right)\left(\gamma_{q}, \gamma_{p} \in \mathbb{N}^{d}\right)$, we have to consider

$$
\xi^{\gamma} \omega(\eta-\xi, \eta)^{J}=\sum_{\gamma_{1}+\gamma_{2}=\gamma} \sum_{|\beta|=J}(-1)^{\left|\beta_{q}\right|+\left|\gamma_{1}\right|} \frac{\gamma !}{\gamma_{1} ! \gamma_{2} !} \frac{J !}{\beta !}(\eta-\xi)^{\beta+\gamma_{1}} \eta^{\widehat{\beta}+\gamma_{2}}
$$

where $\beta=\left(\beta_{q}, \beta_{p}\right)$ with $\beta_{q}, \beta_{p} \in \mathbb{N}^{d}$. The sum contains at most $C_{d}^{J+|\gamma|}$ terms and since

$$
\frac{J !}{\beta !} \leq(2 d)^{J}, \quad \frac{\gamma !}{\gamma_{1} ! \gamma_{2} !} \leq 2^{|\gamma|}
$$

we conclude that (2.8) is now a simple consequence of the fact that

$$
\int_{\mathcal{M}^{*}}\left|\xi^{\widehat{\alpha}} \mathcal{F} f(\xi)\right| \mathrm{d} \nu(\xi)=\int_{\mathcal{M}^{*}}\left|\mathcal{F} \partial^{\alpha} f(\xi)\right| \mathrm{d} \nu(\xi) \leq C_{d} \sup _{\left|\alpha_{1}\right| \leq 2 d+1}\left\|\partial^{\alpha_{1}+\alpha} f\right\|_{\infty} .
$$

We omit the details.

Remark. The above proof can be repeated verbatim if $\mathcal{M}=\mathbb{R}^{2 d}$ and $\mathcal{B}\left(\mathbb{R}^{2 d}\right)$ is replaced by $\mathcal{S}\left(\mathbb{R}^{2 d}\right)$.

We now present a unified version of Egorov Theorem, that is the semiclassical analysis of $e^{i t O p^{W}(g) / \hbar} O p^{W}(f) e^{-i t O p^{W}(g) / \hbar}$ for $f, g \in \mathcal{B}(\mathcal{M})$, with $g$ real valued. This result is well known for $\mathcal{M}=\mathbb{R}^{2 d}[10,22,16,18$ and the purpose of what follows is essentially to prove a similar result for $\mathcal{M}=\mathbb{T}^{2 d}$, with an explicit remainder term. The result is based on the following simple remark: if $A$ is a bounded self-adjoint operator and $B(t)$ is a strongly $C^{1}$ family of bounded operators, then

$$
e^{i t A / \hbar} B(0) e^{-i t A / \hbar}-B(t)=\frac{i}{\hbar} \int_{0}^{t} e^{i(t-s) A / \hbar}\left(\hbar i \frac{d}{d s} B(s)+[A, B(s)]\right) e^{-i(t-s) A / \hbar} .
$$

We shall use this formula with $A=O p^{W}(g)$ and $B(t)$ of the form

$$
B(t)=\sum_{j<J} \hbar^{j} O p^{W}\left(f_{j}(t)\right)
$$

with $f_{0}(t), \cdots, f_{J-1}(t) \in \mathcal{B}(\mathcal{M})$ such that $\sum_{j<J} \hbar^{j} f_{j}(0)=f\left(i . e . B(0)=O p^{W}(f)\right)$ and

$$
\hbar i \frac{d}{d s} B(s)+[A, B(s)]=\mathcal{O}\left(\hbar^{J+1}\right)
$$

where $\mathcal{O}\left(h^{J+1}\right)$ is to be understood the operator norm on $\mathcal{H}$. Expanding $[A, B(s)]$ in powers of $\hbar$ by means of Proposition 2.1] (2.14) leads to the following conditions on the functions $f_{j}(s)$

$$
\begin{aligned}
& \partial_{s} f_{0}-\left\{g, f_{0}\right\}=0, \quad f_{0}(0)=f, \\
& \partial_{s} f_{j}-\left\{g, f_{j}\right\}=2 i \sum_{l+k=j+1} g \#_{k} f_{l}, \quad f_{j}(0)=0 \text { for } j \geq 1
\end{aligned}
$$


where, in the last sum, $3 \leq k \leq J-l$ is odd and $l \leq J-1$, which implies actually that $l \leq j-2$. This system is thus triangular and can be solved using the Hamiltonian flow $\phi^{s}$ of $g$, since the solution of $\partial_{s} a-\{g, a\}=b$ with $a_{s=0}=a_{0}$ is given by

$$
a(s, x)=a_{0}\left(\phi^{s}(x)\right)+\int_{0}^{s} b\left(\tau, \phi^{s-\tau}(x)\right) \mathrm{d} \tau, \quad x \in \mathcal{M} .
$$

Note that if $\mathcal{M}=\mathbb{T}^{2 d}$ and $g$ is identified with a $\mathbb{Z}^{2 d}$ periodic function on $\mathbb{R}^{2 d}$, the associated Hamiltonian flow $\tilde{\phi}^{s}$ on $\mathbb{R}^{2 d}$ is easily seen to satisfy the identity $\tilde{\phi}^{s}(x+n)=\tilde{\phi}^{s}(x)+n$ for all $x \in \mathbb{R}^{2 d}$ and $n \in \mathbb{Z}^{2 d}$. This shows that the formulas for the $f_{j}(s)$ are the same for $\mathcal{M}=\mathbb{R}^{2 d}$ and $\mathbb{T}^{2 d}$, if $f$ and $g$ are $\mathbb{Z}^{2 d}$ periodic.

Let us now define the linear operators $L_{j}^{s}$ on $\mathcal{B}(\mathcal{M})$ by $L_{j}^{s} f:=f_{j}(s)$. We have

$$
L_{0}^{s} f=f \circ \phi^{s}, \quad L_{j}^{s} \equiv 0 \text { for } j \text { odd }
$$

the latter being a consequence of the (skew) symmetry of $\#_{k}$ for $k$ (odd) even. For $j \geq 2$ even, an induction shows that

$$
\begin{aligned}
L_{j}^{s}=\frac{1}{(2 i)^{j}} & \sum_{k=1}^{j / 2} \sum_{m_{1}+\cdots+m_{k}=j / 2} \sum_{\left|\alpha_{1}+\beta_{1}\right|=1+2 m_{1}} \cdots \sum_{\left|\alpha_{k}+\beta_{k}\right|=1+2 m_{k}} \\
& \int_{0}^{s} \cdots \int_{0}^{s_{k-1}} L_{0}^{s-s_{1}} M^{\alpha_{1}, \beta_{1}} L_{0}^{s_{1}-s_{2}} \cdots M^{\alpha_{k}, \beta_{k}} L_{0}^{s_{k}} \mathrm{~d} s_{k} \cdots \mathrm{d} s_{1}
\end{aligned}
$$

where $m_{1} \geq 1, \cdots, m_{k} \geq 1$ in the sum and $M^{\alpha, \beta}$ is the differential operator

$$
M^{\alpha, \beta}=\frac{(-1)^{|\alpha|}}{\alpha ! \beta !} \partial_{q}^{\beta} \partial_{p}^{\alpha} g \partial_{q}^{\alpha} \partial_{p}^{\beta} .
$$

Taking the remainders into account, one gets the following result:

Theorem 2.2 (Egorov theorem). For all $f, g \in \mathcal{B}(\mathcal{M})$ with $g$ real valued and all $J \geq 1$ we have

$$
e^{i t O p p^{W}(g) / \hbar} O p^{W}(f) e^{-i t O p^{W}(g) / \hbar}=\sum_{j<J} \hbar^{j} O p^{W}\left(L_{j}^{t} f\right)+\hbar^{J} R_{J}^{t}(f, \hbar)
$$

where the operator $R_{J}^{t}(f, \hbar)$ has the following explicit form

$$
i \int_{0}^{t} e^{i(t-s) O p^{W}(g) / \hbar}\left(\sum_{l<J} O p^{W}\left(r_{J-l+1}^{\hbar}\left(g, L_{l}^{s} f\right)\right)-O p^{W}\left(r_{J-l+1}^{\hbar}\left(L_{l}^{s} f, g\right)\right)\right) e^{-i(t-s) O p^{W}(g) / \hbar} \mathrm{d} s .
$$

Note that estimates on $\left\|R_{J}^{t}(f, \hbar)\right\|_{\mathcal{H} \rightarrow \mathcal{H}}$ can then be derived from (2.7), (2.8) and estimates on the derivatives of $L_{j}^{s} f$. This will be extensively used in the next section.

\section{Perturbations of quantized hyperbolic maps}

In this section, we address the problem of the semi-classical approximation of $U_{\epsilon}^{-t} O p^{W}(f) U_{\epsilon}^{t}$ as $\hbar \downarrow 0$ in the Ehrenfest time limit $t \approx|\ln \hbar|$, when $U_{\epsilon}$ is a unitary operator on $\mathcal{H}$ of the form

$$
U_{\epsilon}=e^{-i \epsilon O p^{W}(g) / \hbar} M(A)
$$


with $M(A)$ the quantization of a symplectic matrix with integer entries $A \in S p(d, \mathbb{Z})$. We refer to [7, 4, 5] and 14 for the definition of $M(A)$ by mean of the metaplectic representation of $S p(d, \mathbb{R})$ and only quote the properties that we need. The operator $M(A)$ is defined, up to a phase, as the unique operator on $\mathcal{S}^{\prime}\left(\mathbb{R}^{d}\right)$ such that

$$
M(A)^{-1} O p^{W}(f) M(A)=O p^{W}(f \circ A), \quad \forall f \in \mathcal{B}\left(\mathbb{R}^{2 d}\right) .
$$

If $\mathcal{M}=\mathbb{R}^{2 d}, M(A)$ is unitary on $L^{2}\left(\mathbb{R}^{d}\right)$, but if $\mathcal{M}=\mathbb{T}^{2 d}$ and $\mathcal{H}=\mathcal{H}_{\hbar}(\kappa)$ one has to choose special values of $\kappa$ to ensure that $M(A)$ maps $\mathcal{H}_{\hbar}(\kappa)$ into itself, in which case $M(A)$ is unitary (see [7] for more details); from now on, we shall assume that such a choice, which depends on $\hbar$, has been made. Then, (3.1) holds on $\mathcal{M}=\mathbb{R}^{2 d}$ and $\mathbb{T}^{2 d}$ and this is often expressed by saying that for linear evolutions 'Egorov is exact', meaning there is no remainder term.

Let us now describe the results of this section. We will denote by $\phi^{\epsilon}$ the Hamiltonian flow associated to a fixed real valued $g \in \mathcal{B}(\mathcal{M})$ and consider the discrete group $\left(\Phi_{\epsilon}^{t}\right)_{t \in \mathbb{Z}}$ of symplectomorphisms on $\mathcal{M}$ defined by

$$
\Phi_{\epsilon}=\phi^{\epsilon} \circ A .
$$

Then, by setting

$$
\tilde{L}_{j} f=\left(L_{j}^{\epsilon} f\right) \circ A
$$

with the notations of (2.17) and (2.18), we can consider the functions

$$
\mathcal{L}_{0}^{t} f=f \circ \Phi_{\epsilon}^{t}, \quad \mathcal{L}_{j}^{t} f=\sum_{l_{1}+\cdots+l_{t}=j} \tilde{L}_{l_{1}} \cdots \tilde{L}_{l_{t}} f
$$

defined for $j \geq 1, t \geq 0$ integers and $f \in \mathcal{B}(\mathcal{M})$. Note that they depend on $\epsilon$ but we omit this dependence for notational convenience. Note also that $\mathcal{L}_{0}^{t}=\left(\tilde{L}_{0}\right)^{t}$ and that $\mathcal{L}_{j}^{t} \equiv 0$ if $j$ is odd. Our goal is to show that

$$
U_{\epsilon}^{-t} O p^{W}(f) U_{\epsilon}^{t} \sim \sum h^{j} O p^{W}\left(\mathcal{L}_{j}^{t} f\right), \quad \hbar \downarrow 0,
$$

in a scale of times $t$ described in terms of exponents $\Gamma_{A}, \Gamma_{g}$ that we now define.

For the sake of simplicity, we shall assume that $A$ is diagonalizable over $\mathbb{R}$, meaning that there exists an invertible matrix $P$ with real entries such that $A=P^{-1} D P$ with $D$ diagonal. Note that such a condition is of course satisfied if $A$ is symmetric, e.g. the cat map. At the end of the section, we explain how to cope with general symplectic matrices $A \in S p(d, \mathbb{Z})$. Let us define $\Gamma_{A} \geq 0$ by

$$
e^{\Gamma_{A}}=\sup _{\sigma(A)}|\lambda|
$$

Of course, this quantity is well defined for any invertible matrix $A$ with real or complex spectrum. For $z=\left(z_{1}, \cdots, z_{2 d}\right) \in \mathbb{C}^{2 d}$, we denote by $|z|:=\left(\left|z_{1}\right|^{2}+\cdots\left|z_{2 d}\right|^{2}\right)^{1 / 2}$ its standard hermitian norm and set $\|z\|_{P}:=|P z|$. The interest of the norm $\|\cdot\|_{P}$ is that we have

$$
\|A z\|_{P} \leq e^{\Gamma_{A}}\|z\|_{P}, \quad\|\operatorname{Im} A z\|_{P} \leq e^{\Gamma_{A}}\|\operatorname{Im} z\|_{P} \quad \forall z \in \mathbb{C}^{2 d},
$$

which we shall use extensively in the sequel.

Then, inspired by [23, [8, we define the open sets $\Omega_{\delta} \subset \mathbb{C}^{2 d}$ for $\delta>0$ by

$$
\Omega_{\delta}=\left\{z \in \mathbb{C}^{2 d} \mid\|\operatorname{Im} z\|_{P}<\delta\right\}
$$


and we consider the family of norms $\|.\|_{\tau, \delta}$ defined for $\tau \in(0,1)$ by

$$
\|f\|_{\tau, \delta}=\sup _{z \in \Omega_{\tau \delta}}|f(z)|
$$

for functions $f$ which are bounded and analytic on $\Omega_{\delta}$. We can now set

$$
\Gamma_{g}=\sup _{z \in \Omega_{\delta}}\|\| \mathcal{J} \nabla^{2} g(z) \|_{P}, \quad \mathcal{J}=\left(\begin{array}{cc}
0 & I \\
-I & 0
\end{array}\right)
$$

with $\nabla^{2} g$ the Hessian matrix of $g$ and $\|\mid B\|_{P}:=\sup _{z \neq 0}\|B z\|_{P} /\|z\|_{P}$ for $B \in M_{2 d}(\mathbb{C})$. Note that $\Gamma_{g} \neq 0$ unless $g$ is constant which is a trivial situation. We then define

$$
\Gamma_{\epsilon}=\Gamma_{A}+\epsilon \Gamma_{g}
$$

and our main result is the following:

Theorem 3.1. Assume that $f, g \in \mathcal{B}(\mathcal{M})$, with $g$ real valued, have bounded and analytic extensions to $\Omega_{\delta}$ for some $\delta>0$. Then, for all $0<\nu<2$, there exists $J_{0}>0$ such that for all $J>J_{0}$

$$
U_{\epsilon}^{-t} O p^{W}(f) U_{\epsilon}^{t}=\sum_{j<J} \hbar^{j} O p^{W}\left(\mathcal{L}_{j}^{t} f\right)+\hbar^{J} \varrho_{J}^{t}(f, \epsilon, \hbar)
$$

with a remainder such that, for all $0 \leq \epsilon \leq 1$,

$$
\|\left.\hbar^{J} \varrho_{J}^{t}(f, \epsilon, \hbar)\right|_{\mathcal{H} \rightarrow \mathcal{H}} \rightarrow 0 \quad \text { as } \hbar \rightarrow 0 \quad \text { if } \quad 0 \leq t \leq \frac{2-\nu}{3 \Gamma_{\epsilon}}|\ln \hbar| .
$$

The reader may wonder what (3.5) means if $\Gamma_{\epsilon}=0$. In such a case $\Gamma_{g}=0$ thus $g$ is constant so (3.4) becomes $U_{\epsilon}^{-t} O p^{W}(f) U_{\epsilon}^{t}=O p^{W}\left(f \circ A^{t}\right)$ by (3.1) which holds for all $t \geq 0$. In Section 3, we will anyway be interested in the situation where $\Gamma_{A}>0$ and $\epsilon$ is small so that $\Gamma_{\epsilon}>0$.

We also emphasize that the analyticity assumption is imposed by our need to control high order derivatives of $f$ and $g$ in order to estimate $\varrho_{J}^{t}(f, \epsilon, \hbar)$. Similarly to 8 , we could probably relax such a condition by considering quasi-analytic functions (e.g. Gevrey functions) which would allow us to consider compactly supported $f$.

The rest of this section is now devoted to the proof of Theorem 3.1 The principle is rather simple and is the following: a straightforward application of Theorem 2.2 shows that

$$
U_{\epsilon}^{-1} O p^{W}(f) U_{\epsilon}=\sum_{j<J} \hbar^{j} O p^{W}\left(\tilde{L}_{j} f\right)+\hbar^{J} M(A)^{-1} R_{J}^{\epsilon}(f, \hbar) M(A), \quad \forall J>0,
$$

hence an induction on $t \geq 1$ shows that (3.4) holds with

$$
\varrho_{J}^{t}(f, \epsilon, \hbar)=\sum_{j=J}^{t J} \hbar^{j-J} O p^{W}\left(\mathcal{K}_{j, J}^{t} f\right)+\sum_{s=1}^{t} U_{\epsilon}^{s-t} M(A)^{-1} R_{J}^{\epsilon}\left(\mathcal{E}_{J}^{s-1} f, \hbar\right) M(A) U_{\epsilon}^{t-s}
$$

with the operators $\mathcal{K}_{j, J}^{t}$ and $\mathcal{E}_{J}^{t}$ defined by

$$
\mathcal{K}_{j, J}^{t}=\sum_{\substack{l_{1}+\cdots+l_{t}=j \\ l_{1}<J, \cdots, l_{t}<J}} \tilde{L}_{l_{t}} \cdots \tilde{L}_{l_{1}}, \quad \mathcal{E}_{J}^{t}=\sum_{l_{1}<J} \cdots \sum_{l_{t}<J} \hbar^{l_{1}+\cdots+l_{t}} \tilde{L}_{l_{t}} \cdots \tilde{L}_{l_{1}}
$$

Note that $\mathcal{K}_{j, J}^{t}$ depends on both $j$ and $J$ unless $j<J$ in which case $\mathcal{K}_{j, J}^{t}=\mathcal{L}_{j}^{t}$. Note moreover that $\mathcal{E}_{J}^{t}$ depends on $\hbar$ and that we set $\mathcal{K}_{j, J}^{1}=0, \mathcal{E}_{J}^{0}=\mathrm{id}$.

Thus (2.7) reduces the proof of Theorem 3.1 essentially to estimate the derivatives of $\tilde{L}_{l_{t}} \cdots \tilde{L}_{l_{1}} f$. To that end, we shall use the following extension of a lemma of [8]. 
Lemma 3.2. There exists a constant $C_{P}$ depending only on $P$ such that, if

$$
\|f\|_{\tau, \delta} \leq M\left(\frac{C_{P}}{1-\tau}\right)^{a}, \quad \forall 0<\tau<1
$$

for some $M, a \geq 0$, then for all $\gamma$ we have

$$
\left\|\partial^{\gamma} f\right\|_{\tau, \delta} \leq M(a+|\gamma|) \cdots(a+1) \delta^{-|\gamma|}\left(\frac{C_{P}}{1-\tau}\right)^{a+|\gamma|} \quad \forall 0<\tau<1 .
$$

Proof. In [8], the authors show that the result holds with $P=I$ and $C_{P}=e$. Our lemma follows from their result applied to $f \circ P^{-1}$.

In order to estimate $f \circ \Phi_{\epsilon}$ we will capitalize on two facts: on one hand, 3.3) implies that

$$
\|f \circ A\|_{\tau, e^{-\Gamma_{A} \delta}} \leq\|f\|_{\tau, \delta}
$$

and on the other hand we have, for any $0 \leq s \leq t$,

$$
\|\operatorname{Im} z\|_{P} \leq \tau \delta e^{-t \Gamma_{g}} \Rightarrow\left\|\operatorname{Im} \phi^{t-s}(z)\right\|_{P} \leq \tau \delta e^{-s \Gamma_{g}} .
$$

The latter is actually shown in 8 , only for $P=I$ but the very same method easily leads to this estimate. We therefore omit the proof and rather emphasize that it implies that

$$
\left\|f \circ \phi^{t}\right\|_{\tau, \delta e^{-t \Gamma_{g}}} \leq\|f\|_{\tau, \delta}
$$

which leads to the

Lemma 3.3. Assume that $C_{g}>0$ is such that $\left|\partial^{\gamma} g(z)\right| \leq \gamma ! C_{g}^{|\gamma|}$ for all $z \in \Omega_{\delta}$ and all $|\gamma| \geq 1$. Assume moreover that (3.8) holds. Then for all $j \geq 2$ even and all $s \geq 0$ real, we have

$$
\left\|L_{j}^{s} f\right\|_{\tau, \delta e^{-s \Gamma_{g}}} \leq M\left(\frac{C_{P}}{1-\tau}\right)^{a+\frac{3 j}{2}} e^{\frac{3 j}{2} s \Gamma_{g}}(a+1) \cdots\left(a+\frac{3 j}{2}\right)\left(4 d C_{g} / \delta\right)^{3 j / 2} \sum_{k=1}^{j / 2} \frac{s^{k}}{k !},
$$

for all $\tau \in(0,1)$.

Proof. We first note that, by an easy induction on $k \geq 0$, the following result hods: if $s_{0}, \cdots, s_{k}$ are non negative real numbers such that $s_{0}+\cdots+s_{k}=s$ and $\alpha_{1}, \beta_{1}, \cdots, \alpha_{k}, \beta_{k}$ are non zero multi-indices such that $\left|\alpha_{1}+\beta_{1}\right|+\cdots+\left|\alpha_{k}+\beta_{k}\right|=n$, then for all $\tau \in(0,1)$

$$
\left\|L_{0}^{s_{k}} M^{\alpha_{k}, \beta_{k}} \cdots L_{0}^{s_{1}} M^{\alpha_{1}, \beta_{1}} L_{0}^{s_{0}} f\right\|_{\tau, \delta e^{-s \Gamma_{g}}} \leq M\left(\frac{C_{P}}{1-\tau}\right)^{a+n} C_{g}^{n} \delta^{-n} e^{s n \Gamma_{g}}(a+1) \cdots(a+n) .
$$

This follows from Lemma 3.2 and (3.10) (recall that $M^{\alpha, \beta}$ is defined by (2.19). The lemma is then a consequence of (2.18) combined with the above estimate, the fact that

$$
\begin{aligned}
\#\left\{(\alpha, \beta) \in \mathbb{N}^{2 d}|| \alpha+\beta \mid=1+2 m\right\} & =\frac{(2 d+2 m) !}{(2 d-1) !(2 m+1) !} \leq(2 d)^{1+2 m}, \\
\#\left\{\left(m_{1}, \cdots, m_{k}\right) \in \mathbb{N}^{k} \mid m_{1}+\cdots+m_{k}=j / 2\right\} & =\frac{(k-1+j / 2) !}{(k-1) !(j / 2) !} \leq 2^{k-1+\frac{j}{2}},
\end{aligned}
$$

and the fact that $\int_{0}^{s} \cdots \int_{0}^{s_{k-1}} \mathrm{~d} s_{k} \cdots \mathrm{d} s_{1}=s^{k} / k$ !

We can now state the main ingredient of the proof of Theorem 3.1 
Proposition 3.4. With the same assumptions as in Lemma [3.3, we have: for all $j \geq 2$ and all integers $l_{1}, \cdots, l_{t}$ such that $l_{1}+\cdots+l_{t}=j$, we have for all $\epsilon \in[0,1]$

$$
\left\|\tilde{L}_{l_{t}} \cdots \tilde{L}_{l_{1}} f\right\|_{\tau, \delta e^{-t \Gamma_{\epsilon}}} \leq M\left(\frac{C_{P}}{1-\tau}\right)^{a+\frac{3 j}{2}} e^{\frac{3 t}{2} j \Gamma_{\epsilon}}(a+1) \cdots\left(a+\frac{3 j}{2}\right)\left(4 d C_{g} e^{\epsilon} / \delta\right)^{3 j / 2}
$$

provided that (3.8) holds. In addition, if $|f(z)| \leq M$ on $\Omega_{\delta}$, there exists a constant $K$ such that, for all $t \geq 1$, all $\gamma$ and all $\epsilon \in[0,1]$

$$
\left\|\partial^{\gamma} \mathcal{K}_{j, J}^{t} f\right\|_{\infty} \leq M t^{j} K^{(1+\epsilon) j}(|\gamma|+3 j / 2) ! e^{t \Gamma_{\epsilon}(|\gamma|+3 j / 2)}, \quad 0 \leq j \leq t J .
$$

Proof. Recall that we can assume that $j$ is even. We obtain the first statement by induction on $t \geq 1$ using lemma 3.3 with $s=\epsilon$ and (3.9) which we use through $\|f \circ A\|_{\tau, e^{-3 \Gamma_{A} / 2} \delta} \leq\|f \circ A\|_{\tau, e^{-\Gamma_{A} \delta}} \leq$ $\|f\|_{\tau, \delta}$. This, together with (3.7) then yields the second statement since $\#\left\{l_{1}+\cdots+l_{t}=j\right\} \leq t^{j}$.

Proof of Theorem 3.1, We first estimate $\left\|\sum_{j=J}^{t J} h^{j} O p^{w}\left(\mathcal{K}_{j, J}^{t} f\right)\right\|_{\mathcal{H} \rightarrow \mathcal{H}}$. Using (2.7), (3.11) allows to estimate $\ln \left(\left\|h^{j} O p^{w}\left(\mathcal{K}_{j, J}^{t} f\right)\right\|_{\mathcal{H} \rightarrow \mathcal{H}}\right)$ from above by

$$
j\left\{\ln \hbar+t\left(\frac{3}{2}+\frac{\bar{d}}{J}\right) \Gamma_{\epsilon}+\ln \left(K^{2} t\right)+\left(\frac{3}{2}+\frac{\bar{d}}{J}\right) \ln (\bar{d}+3 j / 2)\right\}+\ln C M
$$

using the fact that $1 / j \leq 1 / J$ and that $\epsilon \in[0,1]$. By choosing $J$ large enough we can assume that

$$
\frac{3}{2}+\frac{\bar{d}}{J} \leq \frac{3}{2-\nu / 2}
$$

Since $j \leq J t$, the term $\ln (\bar{d}+3 j / 2)$ is $\mathcal{O}(\ln |\ln | \hbar||)$ as $\hbar \downarrow 0$ thus we get the existence of a new constant $C$ such that for all $\nu \in(0,2), \hbar \in(0,1], \epsilon \in[0,1], 1 \leq t \leq(2-\nu) / 3 \Gamma_{\epsilon}$ and $j \in[J, t J]$

$$
\left\|h^{j} O p^{w}\left(\mathcal{K}_{j, J}^{t} f\right)\right\|_{\mathcal{H} \rightarrow \mathcal{H}} \leq C \hbar^{\nu j / 2}(\ln (C+|\ln \hbar|))^{C j} \leq \tilde{C} \hbar^{\nu j / 4} .
$$

Since $\sum_{J \leq j \leq t J}$ contains $\mathcal{O}(|\ln \hbar|)$ terms, we see that $\left\|\sum_{j=J}^{t J} h^{j} O p^{w}\left(\mathcal{K}_{j, J}^{t} f\right)\right\|_{\mathcal{H} \rightarrow \mathcal{H}} \rightarrow 0$.

Now the norm of second term of (3.6) multiplied by $\hbar^{J}$ can be estimated by

$$
t J h^{J} \sup _{\substack{0 \leq \tau \leq t-1 \\ s \in[0, \in],}, l<J}\left\|O p^{W}\left(r_{J-l+1}^{\hbar}\left(g, L_{l}^{s} \mathcal{E}_{J}^{\tau} f\right)\right)\right\|_{\mathcal{H} \rightarrow \mathcal{H}}+\left\|O p^{W}\left(r_{J+l-1}^{\hbar}\left(L_{l}^{s} \mathcal{E}_{J}^{\tau} f, g\right)\right)\right\|_{\mathcal{H} \rightarrow \mathcal{H}}
$$

with the notations of Theorem 2.2 We proceed as before to estimate $L_{l}^{s} \mathcal{E}_{J}^{\tau} f$ and we obtain the theorem.

Let us now briefly describe how to prove such results for a general $A \in S p(d, \mathbb{Z})$ with $\Gamma_{A}>0$. We claim that, in this case, we have the following result: for any $\tilde{\Gamma}_{A}>\Gamma_{A}$ there exists an invertible matrix $P$ with real entries such that

$$
\left\|P^{-1} A P\right\| \mid \leq e^{\tilde{\Gamma}_{A}}
$$

where $|\|||| \mid$ is the matrix norm associated to the hermitian norm $|$.$| on \mathbb{R}^{2}$. We can prove this statement as follows. Assume first that the spectrum of $A$ is real and let us choose a basis 
$\left(e_{1}, \cdots, e_{2 d}\right)$ of $\mathbb{R}^{2 d}$ in which $A$ is in Jordan normal form. If $\left(e_{j}, \cdots, e_{j+p}\right)$ corresponds to a Jordan block

$$
J(\lambda)=\left(\begin{array}{ccccc}
\lambda & 1 & 0 & \cdots & 0 \\
0 & \lambda & 1 & \ddots & \vdots \\
\vdots & \ddots & \ddots & \ddots & 0 \\
\vdots & & \ddots & \ddots & 1 \\
0 & \cdots & \cdots & 0 & \lambda
\end{array}\right)
$$

then by changing $\left(e_{j}, e_{j+1}, \cdots, e_{j+p}\right)$ into $\left(e_{j}, \varepsilon e_{j+1}, \cdots, \varepsilon^{p} e_{j+p}\right)$ with $\varepsilon>0$, the above block is changed into the same one with 1 replaced by $\varepsilon$. Proceeding similarly for all the blocks, we obtain the existence of basis in which $A$ is the sum of a diagonal matrix of norm $e^{\Gamma_{A}}$ and of a nilpotent matrix of norm $\mathcal{O}(\varepsilon)$. This leads to the statement when the spectrum is real. For non real eigenvalues $\lambda=\rho e^{i \theta}$, using Jordan normal form over $\mathbb{C}^{2 d}$, we have to consider blocks of the form

$$
\left(\begin{array}{cc}
J(\lambda) & 0 \\
0 & J(\bar{\lambda})
\end{array}\right)
$$

It is then standard that there exists a basis of real vectors in which the endomorphism represented by the above block has a matrix of the form $N+\rho R(\theta)$ where $N$ is nilpotent and $R(\theta)$ is block diagonal matrix of rotations (of dimension 2) of angle $\theta$. Then, by changing this basis as in the case of a real spectrum, we can assume that $N$ is small and we obtain (3.14) in the general case.

\section{Equirepartition of time-evolved localized states}

\subsection{The example of (generalized) coherent states}

In this subsection, we shall prove that the generalized coherent states, defined below, when evolved over sufficiently long times, equidistribute on the torus.

To define the states in question, we proceed as follows. Let

$$
\varphi_{\hbar}(q)=h^{-\mu / 2} \varphi\left(\frac{q}{\hbar^{\mu}}\right)
$$

with $\varphi \in \mathcal{S}\left(\mathbb{R}^{d}\right), \int|\varphi|^{2}=1$ and $\mu \in(0,1)$. Then we set

$$
\varphi_{\hbar}^{a}=U_{\hbar}(a) \varphi_{\hbar}
$$

which defines a family of states in $L^{2}\left(\mathbb{R}^{d}\right)$ indexed by $a \in \mathbb{R}^{2 d}$. These are commonly referred to as (generalized) coherent states. The corresponding states on the torus, i. e. belonging to $\mathcal{H}_{\hbar}(\kappa)$, are defined by

$$
\varphi_{\hbar, \kappa}^{a}:=S_{\hbar}(\kappa) \varphi_{\hbar}^{a}=\left(\sum_{n_{p} \in \mathbb{Z}^{d}} e^{-i \kappa_{q} \cdot n_{p}} U_{\hbar}\left(0, n_{p}\right)\right)\left(\sum_{n_{q} \in \mathbb{Z}^{d}} e^{i \kappa_{p} \cdot n_{q}} U\left(n_{q}, 0\right)\right) \varphi_{\hbar}^{a}
$$

which converges in $\mathcal{S}^{\prime}\left(\mathbb{R}^{d}\right)$ (see [] ). The main property of these states that we shall use is

$$
\left\langle\varphi_{\hbar, \kappa}^{a}, O p^{W}(f) \varphi_{\hbar, \kappa}^{b}\right\rangle_{\mathcal{H}_{\hbar}(\kappa)}=\sum_{n \in \mathbb{Z}^{2 d}}(-1)^{N n_{q} \cdot n_{p}} e^{i \omega(\kappa, n)} e^{i \omega(n, b) / 2 \hbar}\left\langle\varphi_{\hbar}^{a}, O p^{W}(f) \varphi_{\hbar}^{b-n}\right\rangle_{L^{2}\left(\mathbb{R}^{d}\right)}
$$


which is proven in 4]. The best known example of such functions are obtained by choosing $\mu=1 / 2$ and $\varphi(q)=\eta(q):=\pi^{-d / 4} e^{-q^{2} / 2}$. With this choice one obtains the standard coherent states.

If $\tilde{\varphi}$ is another Schwartz function and $\tilde{\varphi}_{\hbar}$ is defined similarly to (4.1), the Wigner function $W_{\hbar}(x)$ associated to $\varphi_{\hbar}, \tilde{\varphi}_{\hbar}$ is defined by

$$
\left\langle\varphi_{\hbar}, O p^{W}(f) \tilde{\varphi}_{\hbar}\right\rangle_{L^{2}\left(\mathbb{R}^{d}\right)}=\int_{\mathbb{R}^{2 d}} f(x) W_{\hbar}(x) \mathrm{d} x
$$

for all $f \in \mathcal{B}\left(\mathbb{R}^{2 d}\right)$. For general $\varphi_{\hbar}, \tilde{\varphi}_{\hbar}$ in $L^{2}\left(\mathbb{R}^{d}\right), W_{\hbar}$ is a distribution, but for Schwartz functions it is a Schwartz function as well given by

$$
W_{\hbar}(x)=(2 \pi \hbar)^{-d} \int e^{-i \tilde{q} \cdot p / \hbar} \overline{\varphi_{\hbar}(q-\tilde{q} / 2)} \tilde{\varphi}_{\hbar}(q+\tilde{q} / 2) \mathrm{d} \tilde{q}, \quad x=(q, p) .
$$

With the simple $\hbar$ dependence considered in (4.1), it is easy to see that the Wigner function $W_{\hbar}^{(a, b)}(x)$ associated to $U_{\hbar}(a) \varphi_{\hbar}$ and $U_{\hbar}(b) \tilde{\varphi}_{\hbar}$ takes the following form for any $a, b \in \mathbb{R}^{2 d}$

$$
W_{\hbar}^{(a, b)}(x)=e^{-i \omega(a, b) / 2 \hbar+i \omega(x, b-a) / \hbar} \hbar^{-d} W_{1}\left(\Sigma_{\hbar}^{\mu}\left(x-\frac{a+b}{2}\right)\right)
$$

where $\Sigma_{\hbar}^{\mu}$ is the linear map on $\mathbb{R}^{2 d}$ defined by $\Sigma_{\hbar}^{\mu}(q, p)=\left(q / \hbar^{\mu}, p / \hbar^{1-\mu}\right)$ and $W_{1}$ the Wigner function of $\varphi, \tilde{\varphi}$. Note that, since $W_{1} \in L^{1}\left(\mathbb{R}^{2 d}\right)$, (4.5) implies that $\left\|W_{\hbar}^{(a, b)}\right\|_{L^{1}}=\left\|W_{1}\right\|_{L^{1}}$ is independent of $\hbar$. Note also that when $\varphi(q)=\tilde{\varphi}(q)=\eta(q)$, one easily checks that

$$
W_{1}(x)=\pi^{-d} e^{-x^{2}}
$$

which makes (4.5) completely explicit in this case.

Our main result is Theorem 4.7 As explained in the introduction, its proof goes in two steps. First we use the Egorov theorem to establish that on a suitable time scale $\left\langle U_{\epsilon}^{t} \varphi_{\hbar, \kappa}^{a}, O p^{W}(f) U_{\epsilon}^{t} \varphi_{\hbar, \kappa}^{a}\right\rangle_{\mathcal{H}_{\hbar}(\kappa)}$ is equivalent to $\left\langle\varphi_{\hbar}^{a}, O p^{W}\left(f \circ \Phi_{\epsilon}^{t}\right) \varphi_{\hbar}^{a}\right\rangle_{L^{2}\left(\mathbb{R}^{d}\right)}$ (Proposition 4.2). Then we use an estimate on the classical evolution (exponential mixing) to control this last term.

As a warm up for the first step, we show for a particularly simple class of states how the Egorov expansion (3.4) can be reduced to the first term.

Proposition 4.1. Let $\Psi_{\hbar} \in \mathcal{H}$ be a family such that there exists $C$ satisfying

$$
\left|\left\langle\Psi_{\hbar}, O p^{W}(f) \Psi_{\hbar}\right\rangle\right| \leq C|| f \|_{\infty}, \quad 0<\hbar \leq 1
$$

for all $f$ in $\mathcal{B}(\mathcal{M})$ having a bounded and analytic continuation to some $\Omega_{\delta}$. Then

$$
\left\langle U_{\epsilon}^{t} \Psi_{\hbar}, O p^{W}(f) U_{\epsilon}^{t} \Psi_{\hbar}\right\rangle_{\mathcal{H}}-\left\langle\Psi_{\hbar}, O p^{W}\left(f \circ \Phi_{\epsilon}^{t}\right) \Psi_{\hbar}\right\rangle_{\mathcal{H}} \rightarrow 0, \quad \hbar \rightarrow 0
$$

provided $0 \leq t \leq(2-\nu)|\ln \hbar| / 3 \Gamma_{\epsilon}$ for some $\nu \in(0,2)$.

Proof. Using Theorem 3.1 we only have to show that for all $1 \leq j<J$ we have

$$
\hbar^{j}\left\langle\Psi_{\hbar}, O p^{W}\left(\mathcal{L}_{j}^{t} f\right) \Psi_{\hbar}\right\rangle_{\mathcal{H}} \rightarrow 0, \quad \hbar \rightarrow 0
$$

in the specified range of times. This readily follows from the fact that

$$
\hbar^{j}|| \mathcal{L}_{j}^{t} f \|_{\infty} \leq C_{j} \hbar^{j}|\ln \hbar|^{j} e^{-j\left(1-\frac{\nu}{2}\right) \ln \hbar}
$$


by estimate (3.11), where one should recall that $\mathcal{L}_{j}^{t}=\mathcal{K}_{j, J}^{t}$ if $j<J$.

The condition (4.7) is for instance satisfied by coherent states, in both cases $\mathcal{M}=\mathbb{R}^{2 d}$ and $\mathbb{T}^{2 d}$. This readily follows from the $\hbar$ independence of $\left\|W_{\hbar}^{(a, a)}\right\|_{L^{1}}$ if $\mathcal{M}=\mathbb{R}^{2 d}$. In case of the torus, it is a simple exercise using the Poisson summation formula. Note also that, if $f$ is periodic (in particular if $\mathcal{M}=\mathbb{T}^{2 d}$, we can get rid of the analyticity of $f$ since it is the uniform limit of a sequence of trigonometric polynomials.

Nevertheless, regarding coherent states on the torus, the above result is not precise enough for our purpose since the term $\left\langle\varphi_{\hbar, \kappa}^{a}, O p^{W}\left(f \circ \Phi_{\epsilon}^{t}\right) \varphi_{\hbar, \kappa}^{a}\right\rangle$ is not very explicit. This is why we give the next proposition whose proof will also be used in the proof of Theorem 4.9

Proposition 4.2. Fix $a \in \mathbb{R}^{2 d}$ and assume that $0<\mu<1$. Then, for all $f \in C^{\infty}\left(\mathbb{T}^{2 d}\right)$, we have

$$
\left\langle U_{\epsilon}^{t} \varphi_{\hbar, \kappa}^{a}, O p^{W}(f) U_{\epsilon}^{t} \varphi_{\hbar, \kappa}^{a}\right\rangle_{\mathcal{H}_{\hbar}(\kappa)}-\left\langle\varphi_{\hbar}^{a}, O p^{W}\left(f \circ \Phi_{\epsilon}^{t}\right) \varphi_{\hbar}^{a}\right\rangle_{L^{2}\left(\mathbb{R}^{d}\right)} \rightarrow 0, \quad \hbar \rightarrow 0
$$

provided $0 \leq t \leq(2-\nu)|\ln \hbar| / 3 \Gamma_{\epsilon}$ for some $\nu \in(0,2)$.

Proof. Let us first note that, by truncating the Fourier series of $f$, there exists a sequence $f_{M}$ of $\mathbb{Z}^{2 d}$ periodic analytic functions such that $f_{M} \rightarrow f$ in $\mathcal{B}\left(\mathbb{T}^{2 d}\right)$. Since $\left\|O p^{W}(f)-O p p^{W}\left(f_{M}\right)\right\|_{\mathcal{H} \rightarrow \mathcal{H}} \rightarrow 0$ and

$$
\left\langle\varphi_{\hbar}^{a}, O p^{W}\left(f \circ \Phi_{\epsilon}^{t}\right) \varphi_{\hbar}^{a}\right\rangle_{L^{2}\left(\mathbb{R}^{d}\right)}-\left\langle\varphi_{\hbar}^{a}, O p^{W}\left(f_{M} \circ \Phi_{\epsilon}^{t}\right) \varphi_{\hbar}^{a}\right\rangle_{L^{2}\left(\mathbb{R}^{d}\right)} \rightarrow 0, \quad M \rightarrow+\infty
$$

uniformly with respect to $t \in \mathbb{R}$ and $\hbar \in(0,1]$ by (4.5), we are left with the case where $f$ is analytic. Then, by Theorem 3.1. we only have to study the difference

$$
\sum_{j<J} \hbar^{j}\left\langle\varphi_{\hbar, \kappa}^{a}, O p^{W}\left(\mathcal{L}_{j}^{t} f\right) \varphi_{\hbar, \kappa}^{a}\right\rangle_{\mathcal{H}_{\hbar}(\kappa)}-\left\langle\varphi_{\hbar}^{a}, O p^{W}\left(f \circ \Phi_{\epsilon}^{t}\right) \varphi_{\hbar}^{a}\right\rangle_{L^{2}\left(\mathbb{R}^{d}\right)},
$$

thus the result will follow from (4.4) if we show that, in the specified range of times,

$$
\begin{aligned}
\sum_{n \neq 0}\left|\left\langle\varphi_{\hbar}^{a}, O p^{W}\left(f \circ \Phi_{\epsilon}^{t}\right) \varphi_{\hbar}^{a-n}\right\rangle_{L^{2}\left(\mathbb{R}^{d}\right)}\right| & \rightarrow 0, \\
\hbar^{j} \sum_{n \in \mathbb{Z}^{2 d}}\left|\left\langle\varphi_{\hbar}^{a}, O p^{W}\left(\mathcal{L}_{j}^{t} f\right) \varphi_{\hbar}^{a-n}\right\rangle_{L^{2}\left(\mathbb{R}^{d}\right)}\right| & \rightarrow 0 \quad j \geq 1 .
\end{aligned}
$$

We first note that the term corresponding to $n=0$ in (4.9) has been studied in the proof of the previous proposition, and its limit is 0 . We may therefore assume that $n \neq 0$ in both sums. Using (4.5), integrations by parts with $\hbar^{2} \Delta_{x} /|n|^{2}$ show that, for all $j \geq 0$ and all $M>0$

$$
\left|\hbar^{j}\left\langle\varphi_{\hbar}^{a}, O p^{w}\left(\mathcal{L}_{j}^{t} f\right) \varphi_{\hbar}^{a-n}\right\rangle_{L^{2}\left(\mathbb{R}^{d}\right)}\right| \leq C_{j}|n|^{-2 M} \sum_{|\gamma| \leq 2 M} \hbar^{j+2 M-\bar{m}(2 M-|\gamma|)}\left\|\partial^{\gamma} \mathcal{L}_{j}^{t} f\right\|_{\infty},
$$

where $\bar{m}=\max (\mu, 1-\mu)$. We get the result by the simple observation that

$$
\hbar^{j+2 M-\bar{m}(2 M-|\gamma|)}\left\|\partial^{\gamma} \mathcal{L}_{j}^{t} f\right\|_{\infty} \leq C_{f} \hbar^{2 \nu j+C M} \rightarrow 0, \quad \hbar \rightarrow 0
$$

for $|\gamma| \leq 2 M$, with $C=2(1+\nu) / 3$ if $\bar{m}<(2-\nu) / 3$ and $C=2(1-\bar{m})$ otherwise. This follows from (3.11) by distinguishing both cases $\bar{m} \geq(2-\nu) / 3$ and $\bar{m}<(2-\nu) / 3$.

This proposition, combined with (4.5) allows us to reduce the study of the matrix elements of evolved coherent states to a problem in classical dynamics. By this, we mean that the main result 
of this section, Theorem 4.7 is a direct consequence of Proposition 4.2 and of the mixing estimates given in the Appendix A.

Note that from now on, we shall be working with $d=1$. As explained in the introduction, the reason for this is that, whereas the mixing rate is controlled by the smallest Lyapounov exponent of $A$, the error in the Egorov theorem is controlled by its largest Lyapounov exponent.

As a warm-up, and in order to bring out the main strategy, we first prove a simplified version of the result:

Theorem 4.3. Assume that $\Gamma_{A}>0$. Let a in $\mathbb{R}^{2}, f \in \mathcal{B}\left(\mathbb{T}^{2}\right)$ and $1 / 3<\mu<2 / 3$. Then, for all $\nu>0$ there exists $\epsilon_{0}$ small enough (independent of $f$ ) such that for $|\epsilon|<\epsilon_{0}$ we have

$$
\left\langle U_{\epsilon}^{t} \varphi_{\hbar, \kappa}^{a}, O p^{W}(f) U_{\epsilon}^{t} \varphi_{\hbar, \kappa}^{a}\right\rangle_{\mathcal{H}_{\hbar}(\kappa)} \rightarrow \int_{\mathbb{T}^{2}} f(x) \mathrm{d} x, \quad \hbar \rightarrow 0
$$

provided that

$$
\frac{\bar{m}+\nu}{\Gamma_{\epsilon}}|\ln \hbar| \leq t \leq \frac{2-\nu}{3 \Gamma_{\epsilon}}|\ln \hbar|, \quad \bar{m}=\max (\mu, 1-\mu) .
$$

Proof. We first remark that, by choosing $0<\Gamma<\Gamma_{A}$ close enough to $\Gamma_{A}$ and $\epsilon$ small enough we have

$$
1>\frac{\Gamma}{\Gamma_{\epsilon}}>\frac{1+\nu / 2}{1+\nu}
$$

Combined with (4.10), this estimate implies that $t /|\ln \hbar|>(\bar{m}+\nu / 2) / \Gamma$ and thus

$$
e^{-t \Gamma} \leq \hbar^{\bar{m}+\frac{\nu}{2}}
$$

By Proposition 4.2 and 4.5 we only have to study the limit of

$$
\int_{\mathbb{R}^{2}}\left(f \circ \Phi_{\epsilon}^{t}\right)(x) W_{\hbar}^{(a, a)}(x) \mathrm{d} x
$$

for which $\int W_{\hbar}^{(a, a)}(x) \mathrm{d} x=1$. Choosing a smooth cutoff function $\chi$ so that $\chi=1$ near 0 and which is supported close to 0 , then setting $g_{\hbar}(x):=W_{\hbar}^{(a, a)}(x) \chi(x-a)$, we have $\left\|W_{\hbar}^{(a, a)}-g_{\hbar}\right\|_{L^{1}}=\mathcal{O}\left(h^{\infty}\right)$ thus

$$
\int_{\mathbb{R}^{2}}\left(f \circ \Phi_{\epsilon}^{t}\right)(x) W_{\hbar}^{(a, a)}(x) \mathrm{d} x-\int_{\mathbb{R}^{2}}\left(f \circ \Phi_{\epsilon}^{t}\right)(x) g_{\hbar}(x) \mathrm{d} x \rightarrow 0, \quad \hbar \downarrow 0
$$

uniformly with respect to $t \in \mathbb{R}$. The last integral can obviously be interpreted as an integral over $\mathbb{T}^{2}$ since $g_{\hbar}$ is supported close to $a$ and consequently we can use Corollary A.2 The result now simply follows from the fact that $e^{-t \Gamma}\left\|g_{\hbar}\right\|_{W^{1,1}}=\mathcal{O}\left(h^{-\bar{m}}\right) e^{-t \Gamma} \rightarrow 0$ by (4.12).

The above proof is a rather direct application of Proposition 4.2 and Corollary $\mathbf{A . 2}$ but it fails if $\bar{m} \geq 2 / 3$ (i.e. $\mu \notin(1 / 3,2 / 3)$ ) since $e^{-t \Gamma} h^{-\bar{m}}>1$, for, in that case, $e^{-t \Gamma}>\hbar^{2 / 3}$. The problem stems from the lower bound in (4.10), which arises because $\nabla W_{\hbar}^{(a, a)}$ behaves like $\hbar^{-\bar{m}}$. One expects on intuitive grounds that it should be possible to replace $\bar{m}$ by $\underline{m}=\min (\mu, 1-\mu)$ which is of course less than $1 / 2$ which is less than $2 / 3$. We shall prove this is true, but for that purpose we will need to exploit some more detailed knowledge about the Anosov diffeomorphisms we study. The trick consists in applying a well known idea in the theory of Anosov systems: it is possible to replace (4.13) by an expression obtained by performing an integral along the stable foliation. 
Since the evolution stretches the function $W_{\hbar}^{(a, a)}$ along the unstable manifold, this corresponds to smoothening out the fastest oscillations in $W_{\hbar}^{(a, a)}$, replacing the latter by a function that has a derivative controlled by $\hbar^{-\underline{m}}$. Let us start the proof. By Proposition 4.2 we have to study (4.13) where $W_{\hbar}^{(a, a)}$ can be replaced, as in the proof of Theorem 4.3 by $g_{\hbar}$ which we can assume to be supported as close to $a$ as we want. This will allow us to use the following result.

Theorem 4.4. [3, 2] For all $\Gamma<\Gamma_{A}$, there exists $\epsilon_{0}$ small enough such that for all $|\epsilon|<\epsilon_{0}$ the following holds: there exist $\sigma_{\epsilon}>0$ and a $C^{1+\sigma_{\epsilon}}$ diffeomorphism $x \mapsto F_{\epsilon}(x)=(s(x), u(x))$, from a neighborhood of $a \in \mathbb{T}^{2}$ to a neighborhood of $0 \in \mathbb{R}^{2}$ such that $F_{\epsilon}(a)=0$ and

$$
\left|\partial_{s}\left(f \circ \Phi_{\epsilon}^{t} \circ F_{\epsilon}^{-1}\right)(u, s)\right| \leq C_{f} e^{-\Gamma t}
$$

for all $t \geq 0$, all $(u, s)$ in the neighborhood of 0 and all $f \in C^{1}\left(\mathbb{T}^{2}, \mathbb{R}\right)$. Here $C^{1+\sigma}$ denotes the corresponding Hölder class.

Using this result, we can perform the following change of variables

$$
\int_{\mathbb{R}^{2}}\left(f \circ \Phi_{\epsilon}^{t}\right)(x) g_{\hbar}(x) \mathrm{d} x=\iint\left(f \circ \Phi_{\epsilon}^{t} \circ F_{\epsilon}^{-1}\right)(u, s)\left(g_{\hbar} \circ F_{\epsilon}^{-1}\right)(u, s) J_{\epsilon}(u, s) \mathrm{d} u \mathrm{~d} s
$$

where $J_{\epsilon} \in C^{\sigma_{\epsilon}}$. On the right hand side of this equation, we eventually want to use Corollary A.2 but the $C^{\sigma_{\epsilon}}$ regularity of $J_{\epsilon}(u, s)$ is not sufficient for that purpose. Fortunately, the term $J_{\epsilon}$ is essentially irrelevant in view of the following result.

Lemma 4.5. i) $\left(g_{\hbar} \circ F_{\epsilon}^{-1}\right)_{0<\hbar \leq 1}$ is a bounded family in $L^{1}\left(\mathbb{R}^{2}\right)$.

ii) For all $\theta \in(0,1)$ there exists a family $J_{\epsilon}^{\hbar}$ such that, if $\|.\|_{\infty}$ is the sup norm over a fixed small neighborhood of 0 ,

$$
\left\|J_{\epsilon}^{\hbar}-J_{\epsilon}\right\|_{\infty} \leq C \hbar^{\sigma_{\epsilon} \theta}, \quad\left\|\nabla J_{\epsilon}^{\hbar}\right\|_{\infty} \leq C \hbar^{-\theta} .
$$

Proof. i) follows from (4.5) and ii) from a standard convolution argument by a $C_{0}^{\infty}$ function $\chi_{\hbar}(u, s)=\hbar^{-2 \theta} \chi\left(u / \hbar^{\theta}, s / \hbar^{\theta}\right)$.

Using this lemma and (4.14), the right hand side of (4.15) takes the form

$$
\int\left(f \circ \Phi_{\epsilon}^{t} \circ F_{\epsilon}^{-1}\right)(u, 0) k_{\hbar}(u) \mathrm{d} u+\mathcal{O}_{1}\left(e^{-\Gamma t}\right)+\mathcal{O}_{2}\left(\hbar^{\sigma_{\epsilon} \theta}\right)
$$

where $\mathcal{O}_{1}$ is uniform with respect to $\hbar \in(0,1], \mathcal{O}_{2}$ is uniform with respect to $t \geq 0$ and where the $C^{1}$ function $k_{\hbar}(u)$ is given by

$$
k_{\hbar}(u)=\int\left(g_{\hbar} \circ F_{\epsilon}^{-1}\right)(u, s) J_{\epsilon}^{\hbar}(u, s) \mathrm{d} s .
$$

Note that $k_{\hbar}$ is bounded in $L^{1}$ and that $\int_{\mathbb{R}} k_{\hbar}(u) \mathrm{d} u \rightarrow 1$ as $\hbar \rightarrow 0$. The key remark is now that the derivative of this function is essentially controlled by $\hbar^{-\underline{m}}$ rather than by $\hbar^{-\bar{m}}$, as a rough estimate would show. That is the content of the following proposition. Note that, in what follows, $q, p$ are the canonical coordinates of $\mathbb{R}^{2}$. They also define local coordinates on $\mathbb{T}^{2}$ close to any $a$, and this makes the following statement clear.

Proposition 4.6. Assume that $\mu \leq 1 / 2$ (i.e. that $\max (\mu, 1-\mu)=1-\mu$ ). Then, if the support of $g_{\hbar}$ is sufficiently close to $a$ and if

$$
\partial_{s}\left(p \circ F_{\epsilon}^{-1}\right)(0,0) \neq 0
$$


then, there exists $\tilde{k}_{\hbar} \in C^{1}(\mathbb{R})$ such that $\left\|k_{\hbar}-\tilde{k}_{\hbar}\right\|_{L^{1}} \rightarrow 0$ as $\hbar \rightarrow 0$ and

$$
\left\|d \tilde{k}_{\hbar} / d u\right\|_{L^{1}} \leq C \hbar^{-\mu}
$$

Proof. The condition (4.16) shows that, if $\delta_{1}, \delta_{2}$ are small enough, $s \mapsto\left(p \circ F_{\epsilon}^{-1}\right)(u, s)$ is a diffeomorphism from $\left(-\delta_{1}, \delta_{1}\right)$ onto its range for each $u \in\left(-\delta_{2}, \delta_{2}\right)$. Thus, if the support of $g_{\hbar}$ is small enough, we can use $\left(p \circ F_{\epsilon}^{-1}\right)(u, s)$ as a new variable in the integral defining $k_{\hbar}$ so that it becomes

$$
k_{\hbar}(u)=\hbar^{-1} \int W_{1}\left(\frac{\tilde{q}(u, p)-q(a)}{\hbar^{\mu}}, \frac{p-p(a)}{\hbar^{1-\mu}}\right) \widetilde{\chi}_{\epsilon}^{\hbar}(u, p) j_{\epsilon}(u, p) \mathrm{d} p
$$

with $j_{\epsilon}(u, p)$ the $C^{\sigma_{\epsilon}}$ jacobian of the change of variable and $\widetilde{\chi}_{\epsilon}^{\hbar}(u, p)$ the term corresponding to $\chi\left(F_{\epsilon}^{-1}(u, s)-a\right) J_{\epsilon}^{\hbar}(u, s)$. Changing again the variable with $\tilde{p}=(p-p(a)) / \hbar^{1-\mu}$, we would get the result if $j_{\epsilon}$ was $C^{1}$, by choosing $\theta=\mu$. We can overcome the non smoothness of $j_{\epsilon}$ by the same principle as for Lemma 4.5 we choose $j_{\epsilon}^{\hbar}$ approaching $j_{\epsilon}$ uniformly on the support of $\tilde{\chi}_{\epsilon}^{\hbar}$, such that $\nabla j_{\epsilon}^{\hbar}=\mathcal{O}\left(\hbar^{-\mu}\right)$ and then

$$
\tilde{k}_{\hbar}(u)=\hbar^{-1} \int W_{1}\left(\frac{\tilde{q}(u, p)-q(a)}{\hbar^{\mu}}, \frac{p-p(a)}{\hbar^{1-\mu}}\right) \tilde{\chi}_{\epsilon}^{\hbar}(u, p) j_{\epsilon}^{\hbar}(u, p) \mathrm{d} p
$$

has the expected properties.

Remark. The condition (4.16) expresses the fact that, at the point $a$, the submanifold $\{q=$ $q(a)\}$ is not aligned with the unstable manifold. Of course, if $\mu>1 / 2$, the same result holds if $\partial_{s}\left(q \circ F_{\epsilon}^{-1}\right)(0,0) \neq 0$.

We are now ready for the proof of the main theorem of this subsection.

Theorem 4.7. Assume that $0<\mu \leq 1 / 3$ (resp. $2 / 3 \leq \mu<1$ ) and that the unstable manifold through $a$ is not aligned with the submanifold $\{q=q(a)\}$ (resp. $\{p=p(a)\})$. Assume moreover that $\Gamma_{A}>0$. Then, there exists $\epsilon_{0}$ such that, for $|\epsilon|<\epsilon_{0}$ and all $f \in C^{\infty}\left(\mathbb{T}^{2}\right)$

$$
\left\langle U_{\epsilon}^{t} \varphi_{\hbar, \kappa}^{a}, O p^{W}(f) U_{\epsilon}^{t} \varphi_{\hbar, \kappa}^{a}\right\rangle_{\mathcal{H}_{\hbar}(\kappa)} \rightarrow \int_{\mathbb{T}^{2}} f(x) \mathrm{d} x, \quad \hbar \rightarrow 0
$$

provided that

$$
\frac{\underline{m}+\nu}{\Gamma_{\epsilon}}|\ln \hbar| \leq t \leq \frac{2-\nu}{3 \Gamma_{\epsilon}}|\ln \hbar|, \quad \underline{m}=\min (\mu, 1-\mu) .
$$

Proof. The above discussion shows that we only have to prove that

$$
\int\left(f \circ \Phi_{\epsilon}^{t} \circ F_{\epsilon}^{-1}\right)(u, 0) \tilde{k}_{\hbar}(u) \mathrm{d} u \rightarrow \int_{\mathbb{T}^{2}} f(x) \mathrm{d} x .
$$

Pick a smooth function $\varrho(s)$ supported close to 0 such that $\int \varrho(s) \mathrm{d} s=1$. Then, using Theorem 4.4 the left hand side of (4.19) takes the form

$$
\iint\left(f \circ \Phi_{\epsilon}^{t} \circ F_{\epsilon}^{-1}\right)(u, s) \tilde{k}_{\hbar}(u) \varrho(s) \mathrm{d} u \mathrm{~d} s+\mathcal{O}\left(e^{-\Gamma_{\epsilon} t}\right)
$$


with $\mathcal{O}\left(e^{-\Gamma_{\epsilon} t}\right)$ uniform with respect to $\hbar \in(0,1]$. This last integral is nothing but

$$
\int_{\mathbb{T}^{2}} f \circ \Phi_{\epsilon}^{t}(x) \tilde{g}_{\hbar}(x) \mathrm{d} x
$$

where $\tilde{g}_{\hbar} \circ F_{\epsilon}^{-1}(u, s)=\tilde{k}_{\hbar}(u) \varrho(s) / J_{\epsilon}(u, s)$. Thus $\tilde{g}_{\hbar}$ is of the form $\tilde{g}_{\hbar}^{(1)} \tilde{g}^{(2)}$ with $\tilde{g}^{(2)} \in C^{\sigma_{\epsilon}}$ independent of $\hbar$ and $\left\|\tilde{g}_{\hbar}^{(1)}\right\|_{L^{1}}+\hbar \underline{\underline{m}}\left\|\nabla \tilde{g}_{\hbar}^{(1)}\right\|_{L^{1}}=\mathcal{O}(1)$. Note also that $\int_{\mathbb{T}^{2}} \tilde{g}_{\hbar} \rightarrow 1$ as $\hbar \rightarrow 0$. Using Lemma 4.5 again to approach $\tilde{g}^{(2)}$ by $C^{1}$ functions, we may assume that $\tilde{g}_{\hbar}$ is $C^{1}$ and satisfies the same bound as $\tilde{g}_{\hbar}^{(1)}$. We can now repeat the arguments of Theorem 4.3 and the result follows.

\subsection{Semiclassical behavior of eigenstates}

We now come to a more general result having applications in the description of the eigenvectors of $U_{\epsilon}$. Assume that $\Psi_{\hbar, \kappa} \in \mathcal{H}_{\hbar}(\kappa)$ satisfies, for all $f \in C^{\infty}\left(\mathbb{T}^{2 d}\right)$,

$$
\left\langle\Psi_{\hbar, \kappa}, O p^{W}(f) \Psi_{\hbar, \kappa}\right\rangle_{\mathcal{H}_{\hbar}(\kappa)} \rightarrow f(0), \quad \hbar \downarrow 0 .
$$

Rather vaguely, this condition says that $\Psi_{\hbar, \kappa}$ is concentrated at 0 . This is confirmed by the following

Lemma 4.8. There exists a sequence of positive numbers $r_{\hbar} \rightarrow 0$ and a family of functions $\chi_{\hbar} \in C^{\infty}\left(\mathbb{T}^{2 d}\right)$ supported in a ball of radius $r_{\hbar}$ centered at 0 (in $\mathbb{T}^{2 d}$ ) such that $0 \leq \chi_{\hbar} \leq 1$ and

$$
\left\|\Psi_{\hbar, \kappa}-(2 \pi \hbar)^{-d} \int_{\mathbb{T}^{2 d}} \chi_{\hbar}(a)\left\langle\eta_{\hbar, \kappa}^{a}, \Psi_{\hbar, \kappa}\right\rangle_{\mathcal{H}_{\hbar}(\kappa)} \eta_{\hbar, \kappa}^{a} \mathrm{~d} a\right\|_{\mathcal{H}_{\hbar}(\kappa)} \rightarrow 0, \quad \hbar \downarrow 0 .
$$

Conversely, if [4.21] holds and $\left\|\Psi_{\hbar, \kappa}\right\|_{\mathcal{H}_{\hbar}(\kappa)} \rightarrow 1$ then [4.20) holds for all $f \in C^{\infty}\left(\mathbb{T}^{2 d}\right)$.

The proof of this lemma is given in Appendix B] where we also recall basic results on the coherent states decomposition over $L^{2}\left(\mathbb{R}^{d}\right)$ and $\mathcal{H}_{\hbar}(\kappa)$. Recall that $\eta_{\hbar, \kappa}^{a}$ is defined by (4.1), (4.2) and (4.3) with $\mu=1 / 2$ and $\eta(q)=\pi^{-d / 4} e^{-q^{2} / 2}$.

The right hand side in (4.20) could of course be replaced by $f\left(a_{0}\right)$ for some $a_{0} \in \mathbb{T}^{2 d}$ or more generally by $\sum_{0 \leq j \leq J} \alpha_{j} f\left(a_{J}\right)$ for finitely many points $a_{0}, \ldots, a_{J}$. Correspondingly, one can then define the concentration on a finite collection of points in a $r_{\hbar}$ neighborhood of those points.

To simplify the notation, we set $\lambda_{\hbar}(a)=\chi_{\hbar}(a)\left\langle\eta_{\hbar, \kappa}^{a}, \Psi_{\hbar, \kappa}\right\rangle_{\mathcal{H}_{\hbar}(\kappa)}$. The above lemma proves that

$$
\psi_{\hbar, \kappa}:=(2 \pi \hbar)^{-d} \int_{\mathbb{T}^{2 d}} \lambda_{\hbar}(a) \eta_{\hbar, \kappa}^{a} \mathrm{~d} a
$$

satisfies (4.20) as well and that

$$
\left\langle\Psi_{\hbar, \kappa}, U_{\epsilon}^{-t} O p^{W}(f) U_{\epsilon}^{t} \Psi_{\hbar, \kappa}\right\rangle_{\mathcal{H}_{\hbar}(\kappa)}-\left\langle\psi_{\hbar, \kappa}, U_{\epsilon}^{-t} O p^{W}(f) U_{\epsilon}^{t} \psi_{\hbar, \kappa}\right\rangle_{\mathcal{H}_{\hbar}(\kappa)} \rightarrow 0, \quad \hbar \downarrow 0
$$

uniformly with respect to $t \geq 0$. This is the first step of the proof of the next theorem, in which the notations $\langle.,$.$\rangle and \|$.$\| stand for \langle., .\rangle_{\mathcal{H}_{\hbar}(\kappa)}$ and $\|.\|_{\mathcal{H}_{\hbar}(\kappa)}$ respectively.

Theorem 4.9. Assume that $\left\|\Psi_{\hbar, \kappa}\right\| \rightarrow 1$ and that (4.21) holds for some sequence $r_{\hbar}$ such that

$$
r_{\hbar} \leq \hbar^{1 / 2-\sigma}
$$


with $\sigma>0$. Then, as $\hbar \rightarrow 0$,

$$
\left\langle\Psi_{\hbar, \kappa}, U_{\epsilon}^{-t} O p^{W}(f) U_{\epsilon}^{t} \Psi_{\hbar, \kappa}\right\rangle-(2 \pi \hbar)^{-2 d} \int_{\mathbb{T}^{2 d}} \int_{\mathbb{T}^{2 d}} \overline{\lambda_{\hbar}(a)} \lambda_{\hbar}(b)\left\langle\eta_{\hbar, \kappa}^{a}, O p^{W}\left(f \circ \Phi_{\epsilon}^{t}\right) \eta_{\hbar, \kappa}^{b}\right\rangle \mathrm{d} a \mathrm{~d} b \rightarrow 0
$$

provided

$$
0 \leq \Gamma_{\epsilon} t \leq\left(\frac{1}{2}+\tau\right)|\ln \hbar|, \quad \frac{1}{2}-3 \tau-4 d \sigma>0 \quad \text { and } \quad \tau<\frac{1}{6} .
$$

If moreover $d=1, \Gamma_{A}>0$ and $\tau-5 \sigma>0$, then there exists $t_{\hbar} \rightarrow \infty$ and $\epsilon(\sigma, \tau)>0$ such that for all $|\epsilon| \leq \epsilon(\sigma, \tau)$

$$
\left\langle\Psi_{\hbar, \kappa}, U_{\epsilon}^{-t_{\hbar}} O p^{W}(f) U_{\epsilon}^{t_{\hbar}} \Psi_{\hbar, \kappa}\right\rangle \rightarrow \int_{\mathbb{T}^{2}} f(x) \mathrm{d} x, \quad \hbar \downarrow 0 .
$$

This theorem generalizes a result of [6], Section 5, where only the case $\epsilon=0$ is treated. The proof is then much simpler, since there is then no error term in the Egorov theorem. The theorem says that, if a sequence of states concentrates sufficiently fast on a point $a$ in $\mathbb{T}^{2}$, then the time evolved states equidistribute on the torus on some logarithmic time scale. Before proving this theorem, we show how it leads to a result on the semiclassical behaviour of the eigenvectors of $U_{\epsilon}$.

Corollary 4.10. Assume that $d=1$ and that $\Gamma_{A}>0$. For any $0<\sigma<1 / 38$, there exists $\epsilon(\sigma)>0$ such that for all $|\epsilon|<\epsilon(\sigma)$, no family $\Psi_{\hbar, \kappa}$ of eigenvectors of $U_{\epsilon}$ can satisfy simultaneously [4.20] for all $f$ and $[4.21]$ with $r_{\hbar} \leq \hbar^{1 / 2-\sigma}$.

We note in passing that a similar result (with a worse value of $\sigma$ ) holds for $d>1$ provided we impose a pinching condition on the Lyapounov exponents of $A$ as mentioned in the introduction.

Roughly speaking, this corollary shows that, if a family of eigenvectors of $U_{\epsilon}$ concentrates on a single point in phase space in the semiclassical limit, then it must do so slowly. In other words, no such sequence can 'live' in a ball of too small a radius $r_{\hbar}$. In view of the comment after Lemma (4.8), it is clear that this result holds also for a pure point measure supported on a finite number of periodic orbits. Given Theorem 4.9 the proof is very simple and identical to the case $\epsilon=0$ treated in [6], Section 5. We repeat it for completeness.

Proof. For any $0<\sigma<1 / 38$, one can find $\tau>5 \sigma$ satisfying (4.22). Furthermore, since $\Psi_{\hbar, \kappa}$ is an eigenfunction, $\left\langle\Psi_{\hbar, \kappa}, U_{\epsilon}^{-t} O p^{W}(f) U_{\epsilon}^{t} \Psi_{\hbar, \kappa}\right\rangle=\left\langle\Psi_{\hbar, \kappa}, O p^{W}(f) \Psi_{\hbar, \kappa}\right\rangle$ for all $t$, thus by choosing $t=t_{\hbar}$ and letting $\hbar \downarrow 0$ we obtain

$$
f(0)=\int_{\mathbb{T}^{2}} f(x) \mathrm{d} x
$$

for all $f \in C^{\infty}\left(\mathbb{T}^{2 d}\right)$, which leads to a contradiction.

Proof of Theorem 4.9. Here again, it is sufficient to assume that $f$ is analytic. Using Theorem 3.1 and Lemma 4.8 it is clear that, if $\tau<1 / 6$ and $t \Gamma_{\epsilon} \leq(1 / 2+\tau)|\ln \hbar|$, we have

$$
\left\langle\Psi_{\hbar, \kappa}, U_{\epsilon}^{-t} O p^{W}(f) U_{\epsilon}^{t} \Psi_{\hbar, \kappa}\right\rangle-\sum_{j<J} \hbar^{j}\left\langle\psi_{\hbar, \kappa}, O p^{W}\left(\mathcal{L}_{j}^{t} f\right) \psi_{\hbar, \kappa}\right\rangle \rightarrow 0, \quad \hbar \downarrow 0 .
$$

The first part of the theorem will thus be proven if we show that, for any $j \geq 2$ (recall that $\mathcal{L}_{j}^{t} \equiv 0$ if $j$ is odd), we have

$$
(2 \pi \hbar)^{-2 d} \hbar^{j} \int_{\mathbb{T}^{2 d}} \int_{\mathbb{T}^{2 d}} \overline{\lambda_{\hbar}(a)} \lambda_{\hbar}(b)\left\langle\eta_{\hbar, \kappa}^{a}, O p^{W}\left(\mathcal{L}_{j}^{t} f\right) \eta_{\hbar, \kappa}^{b}\right\rangle \mathrm{d} a \mathrm{~d} b \rightarrow 0, \quad \hbar \downarrow 0
$$


if $1 / 2-3 \tau-4 d \sigma>0$. Using (4.4) and (4.5), integrations by parts similar to those of proposition 4.2 show easily that, for all $M>0$,

$$
\left\langle\eta_{\hbar, \kappa}^{a}, O p^{W}\left(\mathcal{L}_{j}^{t} f\right) \eta_{\hbar, \kappa}^{b}\right\rangle=\sum_{|n| \leq C}(-1)^{N n_{q} \cdot n_{p}} e^{i \omega(\kappa, n)+i \omega(n, b) / 2 \hbar}\left\langle\eta_{\hbar}^{a}, O p^{W}\left(\mathcal{L}_{j}^{t} f\right) \eta_{\hbar}^{b-n}\right\rangle_{L^{2}}+\mathcal{O}\left(\hbar^{M}\right)
$$

uniformly with respect to $a, b \in[0,1)^{2 d}$ and $\Gamma_{\epsilon} t \leq(1 / 2+\tau)|\ln \hbar|$, with $\tau<1 / 6$. The constant $C$ involved in the sum is such that $|b-n-a| \geq C^{-1}|n|$ for all $a, b \in[0,1)^{2 d}$ and $|n|>C$. On the other hand, using (4.5) and (3.11), one sees that, for any $n \in \mathbb{Z}^{2 d}$ and any $j \geq 2$,

$$
(2 \pi \hbar)^{-2 d} \int_{\mathbb{T}^{2 d}} \int_{\mathbb{T}^{2 d}}\left|\lambda_{\hbar}(a) \lambda_{\hbar}(b)\right| \hbar^{j}\left|\left\langle\eta_{\hbar}^{a}, O p^{W}\left(\mathcal{L}_{j}^{t} f\right) \eta_{\hbar}^{b-n}\right\rangle\right| \mathrm{d} a \mathrm{~d} b \leq C \hbar^{-2 d} r_{\hbar}^{4 d} \hbar^{1 / 2-3 \tau}
$$

since $\left|\lambda_{\hbar}(a)\right| \leq\left\|\Psi_{\hbar, \kappa}\right\|$ is bounded and $\lambda_{\hbar}$ is supported in a set of volume $\mathcal{O}\left(r_{\hbar}^{2 d}\right)$. The first part of the theorem follows.

We now prove the second part. Since $\chi_{\hbar}$ can be chosen of the form $\chi_{\hbar}(a)=\sum_{n_{1} \in \mathbb{Z}^{2 d}} \chi\left(\frac{a+n_{1}}{r_{\hbar}}\right)$ (see the Appendix $\mathbb{B}$ ), it turns out that, for any $M, \chi_{\hbar}(a) \chi_{\hbar}(b)\left\langle\eta_{\hbar, \kappa}^{a}, O p^{W}\left(f \circ \Phi_{\epsilon}^{t}\right) \eta_{\hbar, \kappa}^{b}\right\rangle$ can be written

$$
\sum_{|b-a-n|=\mathcal{O}\left(r_{\hbar}\right)}(-1)^{N n_{q} \cdot n_{p}} e^{i \omega(\kappa, n)+i \omega(n, b) / 2 \hbar}\left\langle\eta_{\hbar}^{a}, O p^{W}\left(f \circ \Phi_{\epsilon}^{t}\right) \eta_{\hbar}^{b-n}\right\rangle_{L^{2}}+\mathcal{O}\left(\hbar^{M}\right)
$$

uniformly with respect to $a, b \in[0,1)^{2 d}$. Now, if $d=1$ and $5 \sigma<\tau$, using (4.5) and proceeding similarly to the proof of Theorem 4.3 we see that for $\epsilon$ small enough and $\Gamma<\Gamma_{A}$ sufficiently close to $\Gamma_{A}$

$$
\left\langle\eta_{\hbar}^{a}, O p^{W}\left(f \circ \Phi_{\epsilon}^{t}\right) \eta_{\hbar}^{b-n}\right\rangle_{L^{2}}-\left\langle\eta_{\hbar}^{a}, \eta_{\hbar}^{b-n}\right\rangle_{L^{2}} \int_{\mathbb{T}^{2}} f(x) \mathrm{d} x=e^{-t \Gamma} \mathcal{O}\left(\hbar^{-1 / 2}+r_{\hbar} / \hbar\right)
$$

uniformly on the set where $|b-n-a|=\mathcal{O}\left(r_{\hbar}\right), a, b \in[0,1)^{2}$. This shows that

$$
(2 \pi \hbar)^{-2} \int_{\mathbb{T}^{2}} \int_{\mathbb{T}^{2}} \overline{\lambda_{\hbar}(a)} \lambda_{\hbar}(b)\left\langle\eta_{\hbar, \kappa}^{a}, O p^{W}\left(f \circ \Phi_{\epsilon}^{t}\right) \eta_{\hbar, \kappa}^{b}\right\rangle \mathrm{d} a \mathrm{~d} b-\left\|\psi_{\hbar, \kappa}\right\|^{2} \int_{\mathbb{T}^{2}} f(x) \mathrm{d} x=\mathcal{O}\left(e^{-t \Gamma} \hbar^{-1 / 2-5 \sigma}\right)
$$

and the result follows.

\section{A A mixing theorem for perturbations of hyperbolic maps on $\mathbb{T}^{2}$.}

Let $A$ be a $2 \times 2$ matrix with integer entries such that $|\operatorname{tr} A|>2$ and $\operatorname{det} A=1$. For notational convenience, we assume that its eigenvalues are positive and we note them $e^{ \pm \Gamma_{A}}$, with $\Gamma_{A}>0$. Let $\phi_{\epsilon}$ be a measure preserving diffeomorphsim on $\mathbb{T}^{2}$, depending on a parameter $\epsilon$, such that

$$
\phi_{\epsilon} \rightarrow \text { id } \quad \text { in } C^{3}\left(\mathbb{T}^{2}\right) \text { as } \epsilon \rightarrow 0 .
$$

We define the associated Ruelle-Perron-Frobenius operator $\mathcal{L}_{\epsilon}$ as the map

$$
\mathcal{L}_{\epsilon} g:=g \circ T_{\epsilon}^{-1}, \quad T_{\epsilon}:=\phi_{\epsilon} \circ A .
$$

Using [3] (more precisely (2.1.7), Example 2.2.6 and Theorem 3) one has the following result. 
Theorem A.1 ([3). For any $\Gamma<\Gamma_{A}$, one can find $\epsilon_{0}>0$ small enough such that the following property holds: for all $|\epsilon| \leq \epsilon_{0}$, there exists a Banach space $\mathcal{B}_{\epsilon}$ of distributions of order 1 , containing $C^{1}\left(\mathbb{T}^{2}\right)$, with norm $\|.\|_{\epsilon}$ such that

$$
\|g\|_{\epsilon} \leq C_{\epsilon}\|g\|_{W^{1,1}}, \quad \forall f \in C^{1}\left(\mathbb{T}^{2}\right)
$$

(with $\|g\|_{W^{1,1}}=\int_{\mathbb{T}^{2}}|g|+\int_{\mathbb{T}^{2}}|\nabla g|$ ) and such that

$$
\mathcal{L}_{\epsilon}=\Pi_{1}+\mathcal{R}_{\epsilon} \quad \text { with } \quad \mathcal{R}_{\epsilon} \Pi_{1}=\Pi_{1} \mathcal{R}_{\epsilon}=0
$$

where $\Pi_{1} g=\langle g, 1\rangle 1$ and $\mathcal{R}_{\epsilon}$ is a bounded operator on $\mathcal{B}_{\epsilon}$ with spectral radius lower than $e^{-\Gamma}$. Here $\langle.,$.$\rangle is the pairing between distributions of order 1$ and $C^{1}$ functions.

As a direct consequence, we obtain

Corollary A.2. For all $\Gamma<\Gamma_{A}$, there exists $\epsilon_{0}$ such that, for all $|\epsilon|<\epsilon_{0}$, one can find $C_{\epsilon, \Gamma}$ satisfying

$$
\left|\int_{\mathbb{T}^{2}} f\left(T_{\epsilon}^{t}(x)\right) g(x) \mathrm{d} x-\int_{\mathbb{T}^{2}} f \int_{\mathbb{T}^{2}} g\right| \leq C_{\epsilon, \Gamma} e^{-t \Gamma}\|f\|_{C^{1}}\|g\|_{W^{1,1}}, \quad \text { for all } f, g \in C^{1}\left(\mathbb{T}^{2}\right), t \geq 0 .
$$

\section{B Generalized coherent states decompositions}

In this appendix, we briefly recall some results on coherent states decompositions as well as some convenient tools for the proof of Lemma 4.8

As it is for instance proven in [14, it is well known that for any $u \in \mathcal{S}\left(\mathbb{R}^{d}\right)$ one has

$$
u=(2 \pi \hbar)^{-d} \int_{\mathbb{R}^{2 d}}\left\langle\varphi_{\hbar}^{a}, u\right\rangle_{L^{2}} \varphi_{\hbar}^{a} \mathrm{~d} a
$$

where $\varphi_{\hbar}^{a}$ is defined by (4.2) with $\mu=1 / 2$. This implies in particular that, for any $\tilde{\varphi} \in \mathcal{S}\left(\mathbb{R}^{d}\right)$,

$$
\|u\|_{L^{2}}^{2}\|\tilde{\varphi}\|_{L^{2}}^{2}=(2 \pi \hbar)^{-d} \int_{\mathbb{R}^{2 d}}\left|\left\langle\tilde{\varphi}_{\hbar}^{a}, u\right\rangle_{L^{2}}\right|^{2} \mathrm{~d} a .
$$

This decomposition on $L^{2}\left(\mathbb{R}^{d}\right)$, known as the coherent states decomposition especially when $\varphi(q)=$ $\eta(q)=\pi^{-d / 4} e^{-q^{2} / 2}$, gives rise to a decomposition on $\mathcal{H}_{\hbar}(\kappa)$

$$
S_{\hbar}(\kappa) u=(2 \pi \hbar)^{-d} \int_{\mathbb{T}^{2 d}}\left\langle\varphi_{\hbar, \kappa}^{a}, S_{\hbar}(\kappa) u\right\rangle_{\mathcal{H}_{\hbar}(\kappa)} \varphi_{\hbar, \kappa}^{a} \mathrm{~d} a,
$$

with the notation of (4.3). This is proven in [7]. Note the important consequence of that formula: for any $\tilde{\varphi} \in \mathcal{S}\left(\mathbb{R}^{d}\right)$

$$
(2 \pi \hbar)^{-d} \int_{\mathbb{T}^{2 d}}\left|\left\langle S_{\hbar}(\kappa) \tilde{\varphi}_{\hbar}^{a}, S_{\hbar}(\kappa) u\right\rangle\right|^{2} \mathrm{~d} a=C_{\tilde{\varphi}}\left\|S_{\hbar}(\kappa) u\right\|_{\mathcal{H}_{\hbar}(\kappa)}^{2}, \quad \forall u \in \mathcal{S}\left(\mathbb{R}^{d}\right) .
$$

These decompositions are particularly convenient since one knows rather precisely the action of pseudodifferential operators on functions of the form (4.2), as we shall see in Lemma B.1 below. Motivated by Lemma 4.8 we shall consider functions $f$ depending possibly on $\hbar$. Let $\varepsilon>0$ and assume that $r_{\hbar}$ is a sequence such that

$$
r_{\hbar} \geq \hbar^{1 / 2-\varepsilon}
$$

and let $f_{\hbar}$ be a family of functions in $\mathcal{B}\left(\mathbb{R}^{2 d}\right)$ such that

$$
\left|\partial^{\gamma} f^{\hbar}(x)\right| \leq C_{\gamma} r_{\hbar}^{-|\gamma|}, \quad x \in \mathbb{R}^{2 d} .
$$


Lemma B.1. There exists a family $P_{\gamma}$ of differential operators with polynomial coefficients (independent of $\hbar$ ) such that for any $f^{\hbar}$ as above and any $M>0$, there exists symbols $f^{(\hbar, M, \gamma)}$ satisfying (B.5) as well and differential operators $Q_{\gamma}^{M}$ with polynomial coefficients (independent of $\hbar$ too) such that

$$
O p^{W}\left(f^{\hbar}\right) U_{\hbar}(a) \varphi_{\hbar}=\sum_{|\gamma|<M} \hbar^{|\gamma| / 2} \partial^{\gamma} f^{\hbar}(a) U_{\hbar}(a)\left(P_{\gamma} \varphi\right)_{\hbar}+\hbar^{M \varepsilon} \sum_{|\gamma| \leq 2 M} O p^{W}\left(f^{\hbar, M, \gamma}\right) U_{\hbar}(a)\left(Q_{\gamma}^{M} \varphi\right)_{\hbar} .
$$

Whenever $A=P_{\gamma}$ or $Q_{\gamma}^{M}$, we have set $(A \varphi)_{\hbar}(q)=h^{-d / 4}(A \varphi)\left(q / \hbar^{1 / 2}\right)$.

Proof. It is essentially standard. Since $U_{\hbar}(-a) O p^{W}(f) U_{\hbar}(a)=O p^{W}(f(.+a))$, we are left with the case $a=0$. Then, the result simply follows by writing the Taylor expansion of $f^{\hbar}$ at 0 and integrating by parts.

Remark. The operators $P_{\gamma}$ can be computed explicitly and in particular $P_{0}=I$.

Combining this result and (4.4), it is not hard to deduce that for any $f^{\hbar} \in C^{\infty}\left(\mathbb{T}^{2 d}\right)$ satisfying (B.5), one has, for all $M>0$,

$$
\left\|O p^{W}\left(f^{\hbar}\right) \varphi_{\hbar, \kappa}^{a}-\sum_{|\gamma|<M} \hbar^{|\gamma| / 2} \partial^{\gamma} f^{\hbar}(a) S_{\hbar}(\kappa) U_{\hbar}(a)\left(P_{\gamma} \varphi\right)_{\hbar}\right\|_{\mathcal{H}_{\hbar}(\kappa)} \leq C \hbar^{M \varepsilon}
$$

uniformly with respect to $a \in[0,1)^{2 d}$. We are now ready for the proof of Lemma 4.8

Proof of Lemma 4.8. We only have to show the existence of a sequence $r_{\hbar} \geq \hbar^{1 / 2-\varepsilon}$ for some $\varepsilon>0$, satisfying $r_{\hbar} \rightarrow 0$, such that, if $0 \leq \chi \leq 1$ is supported close to 0 and $\equiv 1$ near 0 then

$$
\chi_{\hbar}(a):=\sum_{n \in \mathbb{Z}^{2 d}} \chi\left(\frac{a+n}{r_{\hbar}}\right)
$$

will satisfy the result. Let us fix $\varepsilon>0$. Then for any sequence $r_{\hbar} \geq \hbar^{1 / 2-\varepsilon}$, using the Proposition 2.1 one has

$$
O p^{W}\left(1-\chi_{\hbar}\right)^{2}=\sum_{j<M} \hbar^{j} O p^{W}\left(\chi_{j, \hbar}\right)+o(1)
$$

in operator norm, provided $M=M(\varepsilon)$ is large enough. The symbols $\chi_{j, \hbar}$ are such that $\partial^{\gamma} \chi_{j, \hbar}=$ $\mathcal{O}\left(r_{\hbar}^{-|\gamma|-2 j}\right)$ and $\chi_{0, \hbar}=\left(1-\chi_{\hbar}\right)^{2}$, thus using ( $(\underline{B .3})$, (B.4) and (B.6) , one has

$$
\left\|O p^{W}\left(1-\chi_{\hbar}\right) \Psi_{\hbar, \kappa}\right\|^{2}=(2 \pi \hbar)^{-d} \int_{\mathbb{T}^{2 d}}\left(1-\chi_{\hbar}(a)\right)^{2}\left|\left\langle\eta_{\hbar, \kappa}^{a}, \Psi_{\hbar, \kappa}\right\rangle\right|^{2} \mathrm{~d} a+o(1)
$$

using also the fact that $\left\|\Psi_{\hbar, \kappa}\right\| \rightarrow 1$. By Taylor formula, there exists a function $\tilde{\chi} \in C^{\infty}\left(\mathbb{T}^{2 d}\right)$, independent of $\hbar$, such that $\tilde{\chi}(0)=0$ and $\left(1-\chi_{\hbar}(a)\right)^{2} \leq \tilde{\chi}^{2}(a) / r_{\hbar}^{2}$. Since

$$
(2 \pi \hbar)^{-d} \int_{\mathbb{T}^{2 d}} \tilde{\chi}(a)^{2}\left|\left\langle\eta_{\hbar, \kappa}^{a}, \Psi_{\hbar, \kappa}\right\rangle\right|^{2} \mathrm{~d} a \rightarrow 0
$$

by (4.20) applied to $f=\tilde{\chi}^{2}$, we see that $\left\|O p^{W}\left(1-\chi_{\hbar}\right) \Psi_{\hbar, \kappa}\right\| \rightarrow 0$ provided $r_{\hbar}^{2} \rightarrow 0$ more slowly than the left hand side of (B.7). Furthermore there is no restriction to choose $r_{\hbar} \geq \hbar^{1 / 2-\varepsilon}$. Finally, we remark that

$$
O p^{W}\left(\chi_{\hbar}\right) \Psi_{\hbar, \kappa}=(2 \pi \hbar)^{-d} \int_{\mathbb{T}^{2 d}}\left\langle\eta_{\hbar, \kappa}^{a}, \Psi_{\hbar, \kappa}\right\rangle \chi_{\hbar}(a) \eta_{\hbar, \kappa}^{a} \mathrm{~d} a+o(1)
$$


by (B.3), (B.4) and (B.6) again which completes the proof of (4.21). For the converse, we note that

$$
\left\langle\Psi_{\hbar, \kappa}, O p^{W}(f) \Psi_{\hbar, \kappa}\right\rangle-(2 \pi \hbar)^{-d} \int_{\mathbb{T}^{2 d}} \chi_{\hbar}(a)\left\langle\eta_{\hbar, \kappa}^{a}, \Psi_{\hbar, \kappa}\right\rangle\left\langle\Psi_{\hbar, \kappa}, O p^{W}(f) \eta_{\hbar, \kappa}^{a}\right\rangle \mathrm{d} a \rightarrow 0
$$

The result follows then easily from the dominated convergence theorem using (B.6) and (B.4).

\section{References}

[1] N. Anantharaman, The eigenfunctions of the Laplacian do not concentrate on sets of small topological entropy, preprint june 2004.

[2] M. BRIN, G. StuCK, Introduction to dynamical systems, Cambridge Univ. Press (2002).

[3] M. Blank, G. Keller, C. Liverani, Ruelle-Perron-Frobenius spectrum for Anosov maps, Nonlinearity 15, no. 6, 1905-1973 (2002).

[4] F. Bonecchi, S. DE BiÈvre, Exponential mixing and $|\ln \hbar|$ time scales in quantized hyperbolic maps on the torus, Comm. Math. Phys. 211, 659-686 (2000).

[5] Controlling strong scarring for quantized ergodic toral automorphisms, Duke Math. J, Vol. 117, No. 3, 571-587 (2003).

[6] Controlling strong scarring for quantized ergodic toral automorphisms, Section 5, mp_arc 02-81 (2002).

[7] A. Bouzouina, S. DE BiÈvre, Equipartition of the eigenfunctions of quantized ergodic maps on the torus, Comm. Math. Phys. 178, 83-105 (1996).

[8] A. Bouzouina, D. Robert, Uniform semiclassical estimates for the propagation of quantum observables, Duke Math. J, Vol. 111, No. 2, 223-252 (2002).

[9] Y. COLIn DE VERDIÈre, Ergodicité et fonctions propres du Laplacien, Commun. Math. Phys. 102, 497-502 (1985).

[10] Y.V. Egorov, On canonical transformations of pseudo-differential operators (in Russian), Uspekhi Mat. Nauk. 24, no. 5, 235-236 (1969).

[11] F. Faure, S. Nonnenmacher, S. De Bièvre, Scarred eigenstates for quantum cats of minimal periods, Commun. Math. Phys. 239, 449-492 (2003).

[12] F. Faure, S. Nonnenmacher, On the maximal scarring for quantum cat map eigenstates, Commun. Math. Phys. 245, 201-214 (2004).

[13] F. Faure, S. Nonnenmacher, contribution at the Workshop on Random Matrix theory and Arithmetic Aspects of Quantum Chaos, Newton Institute, Cambridge, june 2004.

[14] G.B. Folland, Harmonic analysis in phase space, Ann. Math. Studies, Princeton Univ. Press 122, (1989).

[15] B. Helffer, A. Martinez, D. Robert, Ergodicité et limite semi-classique, Comm. Math. Phys 109, 313-326 (1987). 
[16] L. HöRMANDER, The analysis of linear partial differential operators III, Springer-Verlag (1985).

[17] E. Lindenstrauss, Invariant measures and arithmetic quantum unique ergodicity, Annals of Math., to appear.

[18] D. Robert, Autour de l'approximation semi-classique, Progress in mathematics, 68, Birkhaüser (1987).

[19] Z. Rudnick, P. SARnak, The behaviour of eigenstates of hyperbolic arithmetic manifolds, Commun. Math. Phys. 161, 1, 195-213 (1994).

[20] A. Schnirelman, Ergodic properties of eigenfunctions, Usp. Math. Nauk. 29, 181-182 (1974).

[21] R. Schubert, Semiclassical behaviour of expectation values in time evolved coherent states for large times, preprint january 2004.

[22] M. TAYlor, Pseudo-differential operators, Princeton Mathematical Series 34, Princeton University Press (1981).

[23] F. TRÈves, Introduction to pseudo-differential and Fourier integral operators, Vol. 2: Fourier integral operators, Univ. Ser. Math., Plenum, New-York (1980).

[24] ZELDITCH, Uniform distribution of the eigenfunctions on compact hyperbolic surfaces, Duke Math. J 55, 919-941 (1987). 